Article

\title{
Institutional Ownership and Firm Performance under Stakeholder-Oriented Corporate Governance
}

\author{
Hideaki Sakawa *(1) and Naoki Watanabel(1) \\ Graduate School of Economics, Nagoya City University; Nagoya 4678501, Japan; \\ naoki-watanabe@econ.nagoya-cu.ac.jp \\ * Correspondence: sakawa@econ.nagoya-cu.ac.jp
}

Received: 17 December 2019; Accepted: 28 January 2020; Published: 31 January 2020

check for updates

\begin{abstract}
This study aimed to reveal the role of institutional investors with shareholder-oriented scopes in a stakeholder-oriented economy such as Japan. With financial globalization, the increasing number of institutional shareholders in Japanese corporations enables us to investigate whether their shareholder-oriented perspectives are conducive to taking on effective monitoring roles under stakeholder-oriented corporate governance. This study's sample included large listed firms of the TOPIX 500 in Japan during 2010-2016. Using 2924 firm-year observations, the effect of institutional investors on firm performance was analyzed to test the role of institutional investors in stakeholder-oriented corporate governance. Our study showed that the monitoring role of institutional shareholders, or foreign shareholders, functions effectively in Japanese corporations. In addition, we showed that the monitoring roles of these are expected to strengthen firms through higher growth opportunities. These results implied that institutional shareholders contribute to enhancing sustainable firm performance and constructing sustainable corporate governance mechanisms in a stakeholder-oriented system.
\end{abstract}

Keywords: Agency Theory; institutional shareholder; foreign shareholder; stakeholder-oriented corporate governance

\section{Introduction}

In recent decades, the number of shares owned by institutional shareholders has grown rapidly due to financial globalization [1]. This trend is not only being investigated in countries with shareholder- or market-oriented systems, such as in the United States, but also in countries that have stakeholder-oriented systems, such as Japan. The important monitoring role of institutional shareholders is expected in countries with a dispersed ownership structure, as is the case in the United States [2]. Institutional shareholders are defined as block shareholders who are able to supervise and monitor the firms in which they have ownership. Their monitoring activities can be efficiently performed because they have financial incentives due to their stakes in these companies [3]. In addition, institutional shareholders may have more industry-specific knowledge than smaller shareholders, and this information advantage results in low-cost, effective monitoring [4]. Several studies analyze empirical questions about the monitoring role of institutional ownership (IO) on corporate performance [5].

The presence of institutional shareholders increased under stakeholder-oriented corporate governance in Germany and Japan as part of the process of converging to the Anglo-American corporate governance system in the 1990s [6]. In Japan, institutional investors have begun to take a more active stance only since the early 2000s [7]. First, foreign institutional investors tend to behave as activists through annual general shareholder meetings, and their attitude and presence are enhanced $[7,8]$. Second, domestic institutional shareholders, mainly comprising trust banks, pension 
funds, and non-life insurance companies, tend to have a more performance-oriented scope and seek higher profits than other domestic shareholders [9]. In this sense, foreign and domestic institutional investors are characterized differently from other shareholders in Japanese corporations.

There remain important issues for both academics and practitioners to consider in clarifying the effectiveness of institutional shareholders under a stakeholder-oriented system [10]. From the viewpoint of agency theory, larger institutional shareholdings are expected to improve firm value or performance for two reasons. First, institutional investors effectively take on monitoring roles to mitigate agency and asymmetric information problems [11,12]. In fact, they can encourage managers to avoid under-investment, which can lead to poorer future performance. The second reason is that institutional shareholders actively respond to remove managerial entrenchment devices, such as poison pills [13] Previous studies on institutional investors show other channels through which they improve corporate performance. For example, institutional ownership helps mitigate problems of managerial myopia, where firms tend to avoid investing in longer-term positive net present value projects [14-16].

However, during the 1990s, institutional shareholders were not necessarily expected to be effective for firms under stakeholder-oriented systems. In the early 1990s, foreign ownership in Japan averaged less than 5\% [17]. Japanese corporations tend to be controlled by interlocking ownership structures, known as Keiretsu, and the main banking systems [18]. Under the stakeholder-oriented system, the main banks' monitoring mechanisms were considered standard practice prior to the late 1990s [19]. Following the rescue of the Japanese financial system through government bailouts, the shares of the main banks and their cross-shareholdings were also reduced by the merger and acquisition activity in the 2000s [20]. Monitoring by main banks has weakened following the financial deregulation era [21].

The different perspectives of institutional and domestic shareholders could decrease the effectiveness of monitoring in Japanese firms controlled by stable domestic shareholders. The ratio of foreign ownership in Japanese companies has dramatically increased since the 1990s, and the increased influence of foreign institutional shareholders is expected to improve firm value [22,23]. Foreign investors tend not to favor traditional Japanese management practices [24]. In fact, foreign investors tend to have a negative view of the managerial incentives of diversified Japanese firms, unlike the perspective of domestic shareholders [25].

This study focused on Japanese corporations to examine how institutional shareholders affect corporate performance. While several studies have analyzed the impact of foreign ownership on firm performance [26-28], the effect of institutional investors on firm performance has not been fully explored by previous studies. However, they have highlighted two areas in which the new roles of institutional shareholders are complementary and are replacing the weakened monitoring mechanisms of the main banking institutions. First, foreign shareholdings contribute to reducing information asymmetry among investors [29]. Therefore, institutional shareholders are expected to mitigate agency issues. Second, the rise in shareholder activism in recent decades has been effective in strategically applying pressure to managers in Japanese corporations [30]. These points imply that institutional shareholders have begun to play a role in the Japanese stakeholder-oriented system.

The findings of this study shed light on the Japanese experience of an increase in institutional investors in a stakeholder-oriented system. Specifically, the empirical evidence reveals that institutional investors enhance firm value. Moreover, foreign investors play monitoring roles in firms with higher growth opportunities. In other words, institutional investors are expected to function effectively as monitors in the Japanese stakeholder-oriented system. This suggests that institutional shareholders contribute to generating sustainable returns and promoting economic and corporate governance performance in a stakeholder-oriented system.

The rest of the paper is organized as follows. The next section presents the theoretical framework and research context of the study, and we further develop the hypotheses and present the data and methodology in the third section. The fourth section presents the empirical findings. Next, we discuss our findings in the fifth section. The sixth section offers concluding remarks. 


\section{Theoretical Framework and Institutional Background}

\subsection{Theoretical Framework}

Agency theory presumes that manager-shareholder conflicts result in agency costs, which hurt shareholder value [31]. From the agency theory perspective, a stronger corporate governance mechanism helps mitigate severe agency conflicts. Corporate governance consists of an interrelated system, where some practices will be effective and relevant only in certain combinations, leading to different patterns of corporate governance [32]. In Anglo-Saxon countries, effective monitoring by institutional shareholders with a shareholder-oriented scope is expected in publicly-listed firms [3-5].

In the context of international corporate governance research, shareholder-oriented and stakeholder-oriented systems might be characterized differently [33,34]. Stakeholder-oriented systems are found in economies such as those in Japan and continental European countries [35]. The significant characteristics of stakeholder-oriented corporate governance include internal monitoring systems via concentrated ownership and tight inter-firm relationships that substitute for the effective monitoring mechanisms in shareholder-oriented systems [32]. In addition, shareholders in stakeholder-oriented systems have longer time horizons than those in shareholder-oriented systems [36]. Japanese stakeholder-oriented corporate governance is characterized by business groups with financial, human, and transactional ties and cross-shareholdings [37].

Effective monitoring by institutional shareholders is not expected under stakeholder-oriented corporate governance. By contrast, monitoring in Germany and Japan has been based on relationship-oriented banks rather than active markets for corporate control [19]. Especially in Japan, the long-term nature of bank-firm relationships may also complement a more active role for other stakeholders [38]. The interrelated stable shareholders and bank-firm relationships function as substitutes for institutional shareholder monitoring roles in Japan [6]. In fact, main bank relationships have helped mitigate the accounting conservatism of their client firms [39]. In addition, main bank relationships have effectively functioned to enhance earnings quality [40]. Furthermore, IPO underpricing is mitigated through main bank relationships [41].

Another theoretical perspective relates to the corporate governance convergence of AngloAmerican systems under globalization movements [42]. The globalization of market forces could drive non-Anglo-American countries to make changes in corporate governance in a move toward the Anglo-American model $[26,43,44]$. Using a corporate governance convergence perspective, several studies have examined whether the Anglo-American system is being integrated into Japanese corporate governance, such as through the introduction of committee systems [45] and stock-option based pay [46,47]. The results of these studies imply that Japanese corporate governance is transitioning to the Anglo-American corporate governance system. Therefore, the recent increase in institutional ownership in large Japanese corporations would also be a type of "convergence" of the Anglo-American shareholder-oriented system into a stakeholder-oriented system. From the convergence theory perspective, institutional shareholders would play a complementary role as effective monitors, similar to the interrelated stable shareholders and bank-firm relationships in stakeholder-oriented systems.

\subsection{Research Context}

During the 1990s, Japanese corporate ownership structures featured stable arrangements among Keiretsu memberships, financial institutions, and cross-shareholders [48,49]. After the 2000s, financial deregulation altered the ownership structure of Japanese firms [49]. First, shareholdings of main banks were restricted to $5 \%$. Second, the Japanese financial industry, which was the center of horizontal Keiretsu, underwent consolidation as an effect of significant deficit challenges. For decades, the Japanese corporate governance system has distinctly differed from its Western counterpart [38]. Corporate governance was mainly self-conducted and characterized by large inter-corporate shareholdings and deep involvement of the main financial institutions [50-52]. The group-based and network-oriented corporate governance systems in Japan are characterized as Keiretsu and their cross-shareholdings [53]. 
Cross-shareholders are defined as a system of interlocking share ownership among Japanese companies or banks [54-56]. Since they tend to hold stakes for long periods, Japanese ownership structures were characterized by these stable shareholders before the financial deregulation of the 2000s $[6,19,38]$. After the 2000s, the influence of major banks on Japanese firms began to decline [20].

Since the end of the 1990s, institutional investors, including domestic pension funds and foreign asset managers, began to have a greater presence in Japanese firms [24]. Financial deregulation, or the rescue of the Japanese financial system through government bailouts and loans, was funded by taxpayers, which might have had an effect on stakeholder-oriented governance systems [20]. For example, following financial deregulation, Japanese laws restrict the holdings of financial institutions to less than $5 \%$ of outstanding shares. This restriction directly weakens the main banks' ties. On the other hand, institutional investors have emerged as an influential investor group and have received significant attention as major shareholders. In fact, the increase in foreign investors' holdings in Japanese firms grew from less than 5\% in 1990 to $28 \%$ in 2012 [17].

There have been several subsequent corporate governance reforms in Japan Recent corporate governance reforms in Japan are summarized in Table A2. Following the Abe administration's Japan Revitalization Strategy, Japan's stewardship code was established, which aims to realize the effective monitoring activities of institutional shareholders. Japan's Stewardship Code was revised in 2017. The Financial Service Agency [57] defines "stewardship responsibilities" as the responsibilities of institutional investors to enhance the medium- to long-term investment return for their clients and beneficiaries by improving and fostering the investee companies' corporate value and sustainable growth. In this sense, institutional shareholders in Japan are expected to contribute to increasing corporate value. Furthermore, a corporate governance code was introduced in 2015 for all publicly-listed firms. This code also aims to foster dialogue with shareholders to enhance corporate value and sustainable growth [58], thus providing more focus on the role of institutional shareholders in Japanese corporations.

\section{Hypotheses Development, Data, and Methodology}

\subsection{Hypotheses Development}

\subsubsection{Ownership Monitoring}

Agency theory provides a standard theoretical framework for both academics and practitioners to analyze the conflict between managers and shareholders in a shareholder-oriented system [11]. Given the size of institutional investor equity stakes, they tend to exert considerable pressure on management teams to create wealth for investors [59]. Previous studies have found that institutional investors' active monitoring has been especially effective when amendments to anti-takeover charters have been implemented [60-62].

From an agency perspective, an increase in institutional investors is predicted to contribute to effective monitoring under a stakeholder-oriented system. First, institutional investors help mitigate information asymmetry among investors [29]. This implies that institutional investors help mitigate agency issues such as under-investment problems. Second, shareholder activism can effectively pressure managers in Japanese corporations as needed [30]. As a result, their active engagement in monitoring activities has the potential to enhance firm value. Thus, Hypothesis 1a is proposed:

Hypothesis 1a. Institutional ownership (IO) is positively related to firm value.

Instead of assuming homogeneity of institutional investors, this study analyzed their different characteristics and classified the different types of foreign and domestic institutional investors in Japan. Foreign investors have shareholder-oriented views, unlike domestic investors who have stakeholder-oriented views [17,63]. In addition, firms with a larger percentage of foreign investors tend to support higher long-term incentive structures for executive compensation [46]. This implies 
that foreign shareholders in Japan are incentivized to function as effective monitors. Thus, greater foreign shareholding contributes to increased firm value, as in Hypothesis $1 \mathrm{~b}$.

Hypothesis $\mathbf{1 b}$. Foreign ownership $(F O)$ is positively related to firm value.

\subsubsection{Ownership monitoring in high growth firms}

As stated by [64], firms with better growth opportunities demonstrate better current use of funds than firms without such opportunities. To gauge the interest of institutional investors, this study investigated whether they provide more monitoring to firms with greater growth opportunities. Under a stakeholder-oriented system, corporate managers seek to maximize market share or sales volume rather than short-term profits, which is less common in Anglo-American economies such as the United States $[65,66]$. While Japanese corporations stress growth more than profitability, shareholder-oriented investors, such as foreign investors, place more weight on profitability than on firm growth [26] The views of domestic shareholders, including corporations and commercial banks, might be characterized differently because they have different time horizons. In fact, their investment horizons tend to be longer than 10 years [67]. Therefore, domestic shareholders provide managers with longer decision-making timeframes. Institutional shareholders encourage managers to focus on profitability when their corporations face high growth opportunities. Thus, the monitoring role of institutional shareholders would be more effective for firms with higher growth opportunities.

Hypothesis 2a. The association between institutional ownership (IO) and performance is stronger for firms with higher growth opportunities.

The study's next focus was on the monitoring role of foreign investors for firms with higher growth opportunities. As noted above, foreign shareholders are likely to encourage executives to focus more on profitability [63]. A larger presence of foreign shareholders can prompt corporations to take more risks because they are driven to realize higher growth [68]. Thus, the monitoring and intervention of foreign shareholders who urge managers to seek higher risk/higher return opportunities are characterized differently than those of stable domestic investors. In other words, foreign shareholders are expected to positively strengthen firm value in the face of growth opportunities. This leads to Hypothesis $2 \mathrm{~b}$ :

Hypothesis $\mathbf{2 b}$. The association between foreign ownership (FO) and performance is stronger for firms with higher growth opportunities.

\subsection{Data and Methodology}

This study's sample period was from 2010 to 2016, which is the period following the restructuring of Japanese corporate governance. The sample consisted of publicly-listed firms of the TOPIX 500, excluding financial firms. It comprised 436 firms and 2924 firm-year observations. Financial data for this study were obtained from the Astra Manager database, while data related to ownership structure and board size were obtained from the NPM database and Nikkei NEEDS CGES (Corporate Governance Evaluation Systems).

Following previous studies, the dependent variables were firm performance measures, such as Tobin's $Q$ and ROA (return on assets) [1,51]. Tobin's $Q$ measures the market value of a firm's assets while return on assets (ROA) shows the firm's performance from an accounting perspective. The study first examined the direct relationship between IO and performance in the Japanese context.

To examine the empirical hypotheses, IO variables described by [69] were adopted as the independent variables. The study also focused on the difference between foreign and domestic institutional investors because they might have different views regarding Japanese managers. Following Motta and Uchida [70], IO was divided into foreign shareholdings and domestic shareholdings. Next, the interaction term of ownership and sales growth was added to analyze the moderating effect of 
IO, FO, and domestic institutional ownership (DIO) on the relationship between sales growth and firm performance.

Considering the role of stable shareholders in Japanese corporations, domestic ownership and management shareholdings were included in the regression models. Domestic shareholders were characterized as the main banking relationships for lending and shareholding, while stable ownership was represented as cross-shareholdings and dominant shareholdings [38] Main bank ownership is defined as the amounts of the largest lender's shareholdings for the client firm [38]. Cross-shareholdings are mutual shareholdings among other companies held for long periods, which are regarded as managerial entrenchment devices to protect managers from hostile takeovers and proxy fights. In addition, parent-subsidiary relationships are widely observed in Japan [71,72]. Dominant shareholdings represent the existence of parent firms as the control for the effect of parental control of their publicly-listed subsidiary firms. Parent-subsidiary relationships are criticized by Western media because parent firms might exploit other shareholders [73]. Parent-control of publicly-listed subsidiary firms is examined by [74]. Furthermore, management ownership, consisting of the shareholdings of the board of directors, was used as the control for the relationship between management ownership and firm value [51].

In addition, stable shareholders were used as the control for stakeholder-oriented shareholders. As Aguilera et al. [63] mentioned, stakeholder-oriented shareholders, such as stable shareholders, have different views from other institutional investors Stable shareholders are regarded as "allegiant" shareholders guaranteed to vote with management [75]. "Allegiant" shareholders are "companies, banks, and insurers they do business with, are linked to by history, or have assembled precisely to protect management from any awkward minority shareholder resolutions" [76]. Furthermore, stable shareholdings in Japan function as a management entrenchment device or takeover defense to prevent proxy fights $[75,76]$. Japanese stable shareholders were eager to gain firm-specific information through the connections originating from Keiretsu, financial relationships, or parent-subsidiary relationships.

Several firm characteristics were controlled through variables such as firm size, financial leverage, free cash flow, firm risk, the board size, outside director ratio, a stock option dummy, big auditors dummy (Big N), and the number of analysts. Firm size was measured as the logarithm of firm assets, while financial leverage was used to control for firm capital structure. The firm risk was measured as the standard deviation of stock returns. Free cash flow was used as a measure of the degree of conflicts among shareholders and managers [31], and the number of board members was used to control for board size effects [77] Sakawa and Watanabel [78] show a non-negative relationship between board size and firm value for non-financial industries in Japan. Board independence was controlled as the ratio of outside directors [78]. Stock option dummy was used to control for managerial incentives [46]. The Big N dummy was controlled as a measure of audit quality. Big N equals 1 if a firm is audited by a Big-3 or Big-4 audit firm. Otherwise, it is [40]. Finally, the number of analysts was adopted to control for external monitoring.

The effects of IO, FO, and the other control variables on the dependent variables were examined using a fixed firm effects model because the data contains multiple observations with unobserved heterogeneity per firm [79]. Year dummies were included to capture variations over time. To control for residual dependence, standard errors were clustered at the firm level, and lagged variables were adopted for all the independent and control variables to avoid endogeneity problems.

\section{Estimated Results}

\subsection{Regression Results}

This subsection investigates whether institutional shareholders function well as monitors in the Japanese stakeholder-oriented system. The variable definitions are summarized in Table A1, and the sample descriptive statistics are summarized in Table 1. 
Table 1. Descriptive statistics.

\begin{tabular}{|c|c|c|c|c|c|c|c|c|c|c|c|c|c|c|c|c|c|c|c|c|c|c|}
\hline Variable & Mean & $\begin{array}{l}\text { Standard } \\
\text { Deviation }\end{array}$ & Minimum & Maximum & (1) & (2) & (3) & (4) & (5) & (6) & (7) & (8) & (9) & (10) & (11) & (12) & (13) & (14) & (15) & (16) & (17) & (18) \\
\hline 1. $\mathrm{Q}\left({ }^{*} 100\right)$ & 128.69 & 75.49 & 56.28 & 1353.04 & & & & & & & & & & & & & & & & & & \\
\hline 2. ROA & 6.79 & 6.20 & -17.83 & 101.78 & 0.70 * & & & & & & & & & & & & & & & & & \\
\hline 3. IO & 37.29 & 14.03 & 1.63 & 78.00 & $0.11 *$ & $0.17 *$ & & & & & & & & & & & & & & & & \\
\hline 4. $\mathrm{FO}$ & 25.01 & 12.54 & 0.42 & 92.95 & 0.23 * & $0.30 *$ & $0.76^{*}$ & & & & & & & & & & & & & & & \\
\hline 5. DIO & 12.95 & 6.77 & 0.00 & 40.69 & -0.08 & -0.08 & 0.59 * & 0.02 & & & & & & & & & & & & & & \\
\hline $\begin{array}{c}6 . \\
\text { Management }\end{array}$ & 1.33 & 3.93 & 0.00 & 52.14 & $0.18^{*}$ & $0.27^{*}$ & $\underset{*}{-0.02}$ & 0.03 & $\underset{*}{-0.12}$ & & & & & & & & & & & & & \\
\hline 7. Domestic & 14.62 & 15.13 & 0.00 & 75.74 & $0.08^{*}$ & $0.08^{*}$ & $\underset{*}{-0.39}$ & -0.19 & $\underset{*}{-0.27}$ & $\underset{*}{-0.14}$ & & & & & & & & & & & & \\
\hline 8. Stable & 28.29 & 14.21 & 0.33 & 77.53 & 0.09 * & $0.14 *$ & $\underset{*}{-0.52}$ & -0.28 & -0.37 & 0.11 * & 0.80 * & & & & & & & & & & & \\
\hline 9. Size & 13.28 & 1.18 & 9.44 & 17.68 & -0.27 & -0.38 & 0.09 * & 0.11 * & -0.01 & -0.23 & -0.07 & $\underset{*}{-0.20}$ & & & & & & & & & & \\
\hline 10. HighGS & 0.25 & 0.43 & 0.00 & 1.00 & $0.19 *$ & 0.21 * & $0.11^{*}$ & $0.15^{*}$ & $\underset{*}{-0.04}$ & 0.06 * & -0.02 & -0.02 & -0.05 & & & & & & & & & \\
\hline 11. LowGS & 0.25 & 0.43 & 0.00 & 1.00 & $\underset{*}{-0.08}$ & $\underset{*}{-0.16}$ & 0.03 & -0.03 & $0.11 *$ & -0.03 & 0.01 & -0.01 & 0.01 & $\underset{*}{-0.33}$ & & & & & & & & \\
\hline 12. Leverage & 49.87 & 19.82 & 3.83 & 95.03 & $\underset{*}{-0.24}$ & -0.44 & $\underset{*}{-0.22}$ & -0.28 & 0.03 & -0.17 & -0.03 & $\underset{*}{-0.13}$ & $0.50 *$ & $\underset{*}{-0.07}$ & 0.01 & & & & & & & \\
\hline 13. FreeCash & 2.32 & 5.97 & -45.47 & 55.83 & $0.35 *$ & $0.47^{*}$ & $0.06^{*}$ & 0.15 * & -0.06 & $0.15 *$ & 0.03 & $0.07 *$ & $\underset{*}{-0.21}$ & $0.11^{*}$ & $\begin{array}{c}-0.09 \\
*\end{array}$ & $\underset{*}{-0.21}$ & & & & & & \\
\hline 14. Risk & 2.31 & 0.67 & 0.86 & 5.36 & $\underset{*}{-0.05}$ & $\underset{*}{-0.05}$ & $0.16^{*}$ & $0.04^{*}$ & 0.24 * & 0.00 & 0.03 & 0.00 & $-\underset{*}{-0.04}$ & 0.03 & $0.34^{*}$ & $0.16^{*}$ & 0.00 & & & & & \\
\hline 15. BoardSize & 10.76 & 3.80 & 3.00 & 30.00 & $\underset{*}{-0.11}$ & -0.17 & $\underset{*}{-0.08}$ & $\begin{array}{c}-0.12 \\
*\end{array}$ & 0.01 & $-\underset{*}{-0.16}$ & 0.02 & $\underset{*}{-0.05}$ & $0.35 *$ & $\underset{*}{-0.08}$ & $0.04^{*}$ & 0.20 * & $\begin{array}{c}-0.09 \\
*\end{array}$ & $\begin{array}{c}-0.06 \\
*\end{array}$ & & & & \\
\hline $\begin{array}{c}16 . \\
\text { OutsideDirector }\end{array}$ & 17.08 & 15.41 & 0.00 & 86.67 & $0.09 *$ & 0.02 & $0.16^{*}$ & $0.26^{*}$ & -0.01 & -0.04 & $\underset{*}{-0.07}$ & $\underset{*}{-0.13}$ & $0.16^{*}$ & 0.02 & $\begin{array}{l}-0.05 \\
*\end{array}$ & 0.02 & $0.06^{*}$ & -0.08 & -0.22 & & & \\
\hline $\begin{array}{c}17 . \\
\text { StockOption }\end{array}$ & 0.39 & 0.49 & 0.00 & 1.00 & $0.16^{*}$ & $0.16^{*}$ & $0.19^{*}$ & 0.21 * & $0.06 *$ & $0.08 *$ & -0.02 & -0.03 & -0.04 & $0.04 *$ & $0.04 *$ & -0.10 & 0.08 * & 0.05 * & -0.12 & 0.20 * & & \\
\hline 18. $\mathrm{BigN}$ & 0.90 & 0.30 & 0.00 & 1.00 & -0.01 & -0.03 & $\underset{*}{-0.04}$ & $\begin{array}{c}-0.04 \\
*\end{array}$ & -0.01 & -0.01 & -0.01 & -0.03 & $0.10^{*}$ & -0.01 & 0.00 & $0.12 *$ & 0.01 & 0.03 & -0.06 & $0.14^{*}$ & 0.11 * & \\
\hline 19. Analyst & 2.14 & 0.72 & 0.00 & 3.22 & $0.13 *$ & $0.11 *$ & 0.40 * & $0.39 *$ & $0.16^{*}$ & -0.01 & -0.02 & -0.11 & 0.43 * & $0.10^{*}$ & $0.06 *$ & -0.02 & 0.01 & 0.19 * & $0.07 *$ & 0.16 * & 0.14 * & 0.06 * \\
\hline
\end{tabular}


The results of estimating Tobin's $Q$ are shown in Table 2, with standard errors clustered by firm and year. The coefficients of IO were significantly positive for Models (2) and (3), which supports Hypothesis 1A. Furthermore, FO showed significantly positive results for Models (4) and (5), which was consistent with Hypothesis 1B. This implied that institutional shareholders in Japan are expected to actively act as monitors post-2010. In other words, they might substitute for the monitoring role previously held by the main banks [20]. We also investigated the impact of a one standard deviation increase in IO and FO. First, IO was associated with a $8.19 \%(=14.03 \times 0.58)$ increase in Tobin's Q (column (2)) and was related to a $11.17 \%(=14.03 \times 0.80)$ increase in Tobin's $Q($ Column (3)). Second, FO was associated with a $8.87 \%(=12.54 \times 0.81)$ increase in Tobin's $\mathrm{Q}($ column $(4))$ and is related to a $11.57 \%(=12.54 \times 0.92)$ increase in Tobin's $Q($ Column $(5))$. As for the other control variables, high sales growth and free cash flow were significantly positive for all models. This means that firm value tends to be higher in firms with higher growth opportunities.

Table 2. Estimated results of Tobin's Q.

\begin{tabular}{|c|c|c|c|c|c|}
\hline & (1) & (2) & (3) & (4) & (5) \\
\hline \multicolumn{6}{|c|}{$\mathbf{Q}$} \\
\hline $\mathrm{IO}$ & & $\begin{array}{l}0.584 \text { * } \\
(2.26)\end{array}$ & $\begin{array}{c}0.796^{* *} \\
(2.66)\end{array}$ & & \\
\hline $\mathrm{FO}$ & & & & $\begin{array}{l}0.707 * \\
(2.01)\end{array}$ & $\begin{array}{l}0.923 * \\
(2.45)\end{array}$ \\
\hline DIO & & & & $\begin{array}{l}0.211 \\
(0.56)\end{array}$ & $\begin{array}{l}0.337 \\
(0.83)\end{array}$ \\
\hline Management & $\begin{array}{c}-2.539 * \\
(-1.98)\end{array}$ & $\begin{array}{c}-2.449+ \\
(-1.91)\end{array}$ & & $\begin{array}{c}-2.405+ \\
(-1.86)\end{array}$ & \\
\hline Domestic & $\begin{array}{l}0.113 \\
(0.50)\end{array}$ & $\begin{array}{l}0.209 \\
(0.88)\end{array}$ & & $\begin{array}{l}0.194 \\
(0.81)\end{array}$ & \\
\hline Stable & & & $\begin{array}{c}0.676+ \\
(1.85)\end{array}$ & & $\begin{array}{c}0.615+ \\
(1.68)\end{array}$ \\
\hline Size & $\begin{array}{l}8.681 \\
(0.27)\end{array}$ & $\begin{array}{l}7.200 \\
(0.23)\end{array}$ & $\begin{array}{l}9.472 \\
(0.30)\end{array}$ & $\begin{array}{l}5.211 \\
(0.16)\end{array}$ & $\begin{array}{l}7.000 \\
(0.22)\end{array}$ \\
\hline HighGS & $\begin{array}{l}5.650 * \\
(2.01)\end{array}$ & $\begin{array}{l}5.448 * \\
(1.98)\end{array}$ & $\begin{array}{c}5.142+ \\
(1.91)\end{array}$ & $\begin{array}{l}5.409 * \\
(2.00)\end{array}$ & $\begin{array}{c}5.114+ \\
(1.93)\end{array}$ \\
\hline LowGS & $\begin{array}{l}4.062 \\
(1.53)\end{array}$ & $\begin{array}{l}4.288 \\
(1.64)\end{array}$ & $\begin{array}{c}4.810+ \\
(1.85)\end{array}$ & $\begin{array}{c}4.387+ \\
(1.69)\end{array}$ & $\begin{array}{c}4.924+ \\
(1.91)\end{array}$ \\
\hline Leverage & $\begin{array}{l}-0.415 \\
(-0.68)\end{array}$ & $\begin{array}{l}-0.344 \\
(-0.55)\end{array}$ & $\begin{array}{l}-0.294 \\
(-0.47)\end{array}$ & $\begin{array}{l}-0.317 \\
(-0.51)\end{array}$ & $\begin{array}{l}-0.265 \\
(-0.43)\end{array}$ \\
\hline FreeCash & $\begin{array}{c}0.414^{* *} \\
(3.28)\end{array}$ & $\begin{array}{c}0.379 \text { ** } \\
(3.07)\end{array}$ & $\begin{array}{c}0.387^{* *} \\
(3.08)\end{array}$ & $\begin{array}{c}0.383^{* *} \\
(3.09)\end{array}$ & $\begin{array}{c}0.393^{* *} \\
(3.11)\end{array}$ \\
\hline Risk & $\begin{array}{c}8.267^{*} \\
(2.12)\end{array}$ & $\begin{array}{l}7.628 \\
(1.92)\end{array}$ & $\begin{array}{c}6.928+ \\
(1.69)\end{array}$ & $\begin{array}{c}7.506+ \\
(1.87)\end{array}$ & $\begin{array}{c}6.844+ \\
(1.66)\end{array}$ \\
\hline BoardSize & $\begin{array}{l}0.098 \\
(0.19)\end{array}$ & $\begin{array}{l}0.072 \\
(0.14)\end{array}$ & $\begin{array}{l}0.056 \\
(0.10)\end{array}$ & $\begin{array}{l}0.076 \\
(0.14)\end{array}$ & $\begin{array}{l}0.061 \\
(0.11)\end{array}$ \\
\hline $\begin{array}{l}\text { Outside } \\
\text { Directors }\end{array}$ & $\begin{array}{l}-0.180 \\
(-1.17)\end{array}$ & $\begin{array}{l}-0.188 \\
(-1.22)\end{array}$ & $\begin{array}{l}-0.160 \\
(-1.04)\end{array}$ & $\begin{array}{l}-0.200 \\
(-1.29)\end{array}$ & $\begin{array}{l}-0.175 \\
(-1.13)\end{array}$ \\
\hline StockOption & $\begin{array}{l}-0.968 \\
(-0.32)\end{array}$ & $\begin{array}{l}-0.556 \\
(-0.18)\end{array}$ & $\begin{array}{l}-0.664 \\
(-0.21)\end{array}$ & $\begin{array}{l}-0.503 \\
(-0.16)\end{array}$ & $\begin{array}{l}-0.603 \\
(-0.19)\end{array}$ \\
\hline BigN & $\begin{array}{c}11.230 * \\
(2.10)\end{array}$ & $\begin{array}{c}10.230+ \\
(1.89)\end{array}$ & $\begin{array}{c}11.860 * \\
(2.14)\end{array}$ & $\begin{array}{c}10.760+ \\
(1.91)\end{array}$ & $\begin{array}{c}12.420 \text { * } \\
(2.17)\end{array}$ \\
\hline Analyst & $\begin{array}{c}11.390^{* * *} \\
(4.04)\end{array}$ & $\begin{array}{c}10.560 * * \\
(3.83)\end{array}$ & $\begin{array}{c}11.250 * * \\
(3.93)\end{array}$ & $\begin{array}{c}10.620^{* *} \\
(3.85)\end{array}$ & $\begin{array}{c}11.260^{* *} \\
(3.95)\end{array}$ \\
\hline Constant & $\begin{array}{c}-5.78 \\
(-0.01)\end{array}$ & $\begin{array}{l}-10.06 \\
(-0.02)\end{array}$ & $\begin{array}{l}-71.40 \\
(-0.18)\end{array}$ & $\begin{array}{l}15.97 \\
(0.04)\end{array}$ & $\begin{array}{l}-36.65 \\
(-0.09)\end{array}$ \\
\hline Number & 2924 & 2924 & 2924 & 2924 & 2924 \\
\hline AdjustedR2 & 0.113 & 0.117 & 0.115 & 0.117 & 0.114 \\
\hline R2within & 0.119 & 0.123 & 0.120 & 0.123 & 0.120 \\
\hline
\end{tabular}


Table 2. Cont.

\begin{tabular}{cccccc}
\hline \multicolumn{7}{c}{$(\mathbf{1})$} & $\mathbf{( 2 )}$ & $\mathbf{( 3 )}$ & $\mathbf{( 4 )}$ & $\mathbf{( 5 )}$ \\
\hline \multicolumn{7}{c}{$\mathbf{Q}$} \\
R2overall & 0.000 & 0.004 & 0.019 & 0.016 & 0.044 \\
R2between & 0.004 & 0.000 & 0.009 & 0.007 & 0.039 \\
$F$ & $9.56^{* *}$ & $9.82^{* *}$ & $10.21^{* *}$ & $9.50^{* *}$ & $9.83^{* *}$ \\
\hline
\end{tabular}

Note: Observations $=2924$. Standard errors are clustered at the firm level. $t$-values are presented in parentheses. See Table A1 for variable definitions. The results of firm dummies and year dummies are not reported. $+p<0.10$, ${ }^{*} p<0.05,{ }^{* *} p<0.01$.

Table 3 presents the relationship between institutional shareholders and firm performance, measured as ROA. The results of Models (2) and (3) provided significantly positive results for IO, consistent with Hypothesis 1A. Next, the coefficients of both FO and DIO were significantly positive in Models (4) and (5). These findings imply that institutional investors and foreign investors enhance firm value, which is consistent with their shareholder-oriented views.

Table 3. Estimated results of ROA (return on assets).

\begin{tabular}{|c|c|c|c|c|c|}
\hline & (1) & (2) & (3) & (4) & (5) \\
\hline & \multicolumn{5}{|c|}{ ROA } \\
\hline $\mathrm{IO}$ & & $\begin{array}{c}0.123^{* *} \\
(3.63)\end{array}$ & $\begin{array}{c}0.148^{* *} \\
(3.71)\end{array}$ & & \\
\hline FO & & & & $\begin{array}{c}0.154^{* *} \\
(3.40)\end{array}$ & $\begin{array}{c}0.178^{* *} \\
(3.50)\end{array}$ \\
\hline $\mathrm{DIO}$ & & & & $\begin{array}{c}0.091 \text { ** } \\
(2.75)\end{array}$ & $\begin{array}{c}0.109 * * \\
(2.95)\end{array}$ \\
\hline Management & $\begin{array}{l}0.004 \\
(0.05)\end{array}$ & $\begin{array}{l}0.023 \\
(0.24)\end{array}$ & & $\begin{array}{l}0.031 \\
(0.32)\end{array}$ & \\
\hline Domestic & $\begin{array}{l}-0.008 \\
(-0.55)\end{array}$ & $\begin{array}{l}0.012 \\
(0.71)\end{array}$ & & $\begin{array}{l}0.012 \\
(0.70)\end{array}$ & \\
\hline Stable & & & $\begin{array}{l}0.075 \text { * } \\
(2.17)\end{array}$ & & $\begin{array}{l}0.071 \text { * } \\
(2.10)\end{array}$ \\
\hline Size & $\begin{array}{c}-6.998^{* *} \\
(-2.69)\end{array}$ & $\begin{array}{c}-7.310 \text { ** } \\
(-2.79)\end{array}$ & $\begin{array}{c}-7.416^{* *} \\
(-2.79)\end{array}$ & $\begin{array}{c}-7.676^{* *} \\
(-2.86)\end{array}$ & $\begin{array}{c}-7.826^{* *} \\
(-2.87)\end{array}$ \\
\hline HighGS & $\begin{array}{c}1.033 \text { ** } \\
(3.65)\end{array}$ & $\begin{array}{c}0.991 * * \\
(3.69)\end{array}$ & $\begin{array}{c}0.985^{* *} \\
(3.69)\end{array}$ & $\begin{array}{c}0.984 \text { ** } \\
(3.72)\end{array}$ & $\begin{array}{c}0.980 * * \\
(3.73)\end{array}$ \\
\hline LowGS & $\begin{array}{c}-1.122 * * \\
(-4.18)\end{array}$ & $\begin{array}{c}-1.075 * * \\
(-4.22)\end{array}$ & $\begin{array}{c}-1.080^{* *} \\
(-4.12)\end{array}$ & $\begin{array}{c}-1.049 * * \\
(-4.23)\end{array}$ & $\begin{array}{c}-1.053^{* *} \\
(-4.14)\end{array}$ \\
\hline Leverage & $\begin{array}{l}0.011 \\
(0.29)\end{array}$ & $\begin{array}{l}0.026 \\
(0.65)\end{array}$ & $\begin{array}{l}0.031 \\
(0.77)\end{array}$ & $\begin{array}{l}0.032 \\
(0.79)\end{array}$ & $\begin{array}{l}0.037 \\
(0.91)\end{array}$ \\
\hline FreeCash & $\begin{array}{c}0.097^{* *} \\
(4.92)\end{array}$ & $\begin{array}{c}0.089^{* *} \\
(5.00)\end{array}$ & $\begin{array}{c}0.089^{* *} \\
(5.09)\end{array}$ & $\begin{array}{c}0.089^{* *} \\
(5.15)\end{array}$ & $\begin{array}{c}0.089^{* *} \\
(5.25)\end{array}$ \\
\hline Risk & $\begin{array}{l}0.213 \\
(0.60)\end{array}$ & $\begin{array}{l}0.079 \\
(0.21)\end{array}$ & $\begin{array}{l}-0.018 \\
(-0.04)\end{array}$ & $\begin{array}{l}0.042 \\
(0.11)\end{array}$ & $\begin{array}{l}-0.051 \\
(-0.13)\end{array}$ \\
\hline BoardSize & $\begin{array}{l}-0.036 \\
(-0.95)\end{array}$ & $\begin{array}{l}-0.041 \\
(-1.14)\end{array}$ & $\begin{array}{l}-0.040 \\
(-1.10)\end{array}$ & $\begin{array}{l}-0.042 \\
(-1.15)\end{array}$ & $\begin{array}{l}-0.041 \\
(-1.11)\end{array}$ \\
\hline $\begin{array}{l}\text { Outside } \\
\text { Directors }\end{array}$ & $\begin{array}{c}-0.030+ \\
(-1.71)\end{array}$ & $\begin{array}{c}-0.032+ \\
(-1.82)\end{array}$ & $\begin{array}{c}-0.030+ \\
(-1.76)\end{array}$ & $\begin{array}{c}-0.034+ \\
(-1.89)\end{array}$ & $\begin{array}{c}-0.033+ \\
(-1.85)\end{array}$ \\
\hline StockOption & $\begin{array}{c}-0.787^{*} \\
(-2.02)\end{array}$ & $\begin{array}{c}-0.700+ \\
(-1.80)\end{array}$ & $\begin{array}{c}-0.706+ \\
(-1.81)\end{array}$ & $\begin{array}{c}-0.688+ \\
(-1.76)\end{array}$ & $\begin{array}{c}-0.695+ \\
(-1.77)\end{array}$ \\
\hline BigN & $\begin{array}{l}-0.290 \\
(-0.60)\end{array}$ & $\begin{array}{l}-0.501 \\
(-1.01)\end{array}$ & $\begin{array}{l}-0.442 \\
(-0.86)\end{array}$ & $\begin{array}{l}-0.462 \\
(-0.92)\end{array}$ & $\begin{array}{l}-0.402 \\
(-0.78)\end{array}$ \\
\hline Analyst & $\begin{array}{c}1.268^{* *} \\
(4.40)\end{array}$ & $\begin{array}{c}1.094^{* *} \\
(3.46)\end{array}$ & $\begin{array}{c}1.147^{* *} \\
(3.67)\end{array}$ & $\begin{array}{c}1.079 * * \\
(3.38)\end{array}$ & $\begin{array}{c}1.131^{* *} \\
(3.59)\end{array}$ \\
\hline Constant & $\begin{array}{c}98.98^{* *} \\
(2.88)\end{array}$ & $\begin{array}{c}98.08^{* *} \\
(2.91)\end{array}$ & $\begin{array}{c}96.41^{* *} \\
(2.87)\end{array}$ & $\begin{array}{c}102.20^{* *} \\
(2.98)\end{array}$ & $\begin{array}{c}101.30^{* *} \\
(2.97)\end{array}$ \\
\hline
\end{tabular}


Table 3. Cont.

\begin{tabular}{cccccc}
\hline & (1) & (2) & (3) & (4) & (5) \\
\hline Number & 2924 & 2924 & 2924 & 2924 & 2924 \\
AdjustedR2 & 0.183 & 0.203 & 0.208 & 0.206 & 0.211 \\
R2within & 0.189 & 0.208 & 0.213 & 0.212 & 0.216 \\
R2overall & 0.196 & 0.215 & 0.224 & 0.232 & 0.241 \\
R2between & 0.226 & 0.244 & 0.257 & 0.272 & 0.284 \\
$F$ & $12.26^{* *}$ & $12.14^{* *}$ & $12.67^{* *}$ & $11.63^{* *}$ & $12.06^{* *}$ \\
\hline
\end{tabular}

Note: Observations $=2924$. Standard errors are clustered at the firm level. $t$-values are presented in parentheses. See Table A1 for variable definitions. The results of firm dummies and year dummies are not reported. $+p<0.10$, ${ }^{*} p<0.05,{ }^{* *} p<0.01$.

The results of including the interaction terms of the ownership variables and the high sales growth dummy are presented in Table 4. Using the results of the four models, both IO and FO show significantly positive results, consistent with Hypotheses 1A and 1B. As for the interaction terms of IO and the high growth rate of sales (High SG), the results were significantly positive for all models. The coefficients of the interaction terms of FO and High SG are also significantly positive. These results supported Hypotheses $2 \mathrm{a}$ and $2 \mathrm{~b}$ and implied that institutional shareholders strengthen monitoring in firms with higher sales growth.

We examined the impact of a one standard deviation increase in $\mathrm{IO}$ and $\mathrm{FO}$ to consider economic significance. First, IO was associated with a $8.64 \%(=14.03 \times 0.62)$ increase in Tobin's Q (column (2)). In addition, $\mathrm{IO}$ was associated with a $1.66 \%(=14.03 \times 0.12)$ increase in ROA (column $(5))$ and is related to a $2.02 \%(=14.03 \times 0.14)$ increase in ROA (Column (5)). Second, FO was related to $9.14 \%(=12.54 \times$ $0.73)$ increase in Tobin's $Q($ Column (4)). Furthermore, FO was associated with a $1.86 \%(=12.54 \times 0.15)$ increase in ROA (column (7)) and was related to a $2.17 \%(=12.54 \times 0.17)$ increase in ROA (Column (8)).

Table 4. Interaction effects of institutional ownership (IO) and growth opportunities on firm performance.

\begin{tabular}{|c|c|c|c|c|c|c|c|c|}
\hline & (1) & (2) & (3) & (4) & (5) & (6) & (7) & (8) \\
\hline & \multicolumn{4}{|c|}{$\mathbf{Q}$} & \multicolumn{4}{|c|}{ ROA } \\
\hline IO & $\begin{array}{l}0.405 \\
(1.56)\end{array}$ & $\begin{array}{l}0.616^{*} \\
(2.04)\end{array}$ & & & $\begin{array}{c}0.118^{* *} \\
(3.59)\end{array}$ & $\begin{array}{c}0.144^{* *} \\
(3.68)\end{array}$ & & \\
\hline $\mathrm{IO}$ & $0.385^{*}$ & $0.402 *$ & & & $0.027^{*}$ & $0.027^{*}$ & & \\
\hline *HighGS & $(2.26)$ & $(2.30)$ & & & $(2.12)$ & $(2.12)$ & & \\
\hline $\mathrm{IO}$ & $0.198+$ & 0.177 & & & -0.008 & -0.011 & & \\
\hline *LowGS & $(1.67)$ & $(1.46)$ & & & $(-0.80)$ & $(-1.03)$ & & \\
\hline FO & & & $\begin{array}{l}0.514 \\
(1.55)\end{array}$ & $\begin{array}{c}0.729 * \\
(2.04)\end{array}$ & & & $\begin{array}{c}0.148^{* *} \\
(3.36)\end{array}$ & $\begin{array}{c}0.173 * * \\
(3.47)\end{array}$ \\
\hline $\mathrm{FO}$ & & & $0.522 * *$ & $0.550^{* *}$ & & & $0.026+$ & $0.026+$ \\
\hline${ }^{*}$ HighGS & & & $(2.64)$ & $(2.80)$ & & & $(1.76)$ & $(1.84)$ \\
\hline FO & & & 0.057 & 0.033 & & & -0.012 & -0.014 \\
\hline *LowGS & & & $(0.43)$ & $(0.25)$ & & & $(-0.77)$ & $(-0.84)$ \\
\hline DIO & & & 0.046 & 0.181 & & & $0.088^{* *}$ & $0.108^{* *}$ \\
\hline DIO & & & -0.204 & -0.227 & & & -0.013 & -0.015 \\
\hline${ }^{*}$ HighGS & & & $(-0.45)$ & $(-0.50)$ & & & $(-0.51)$ & $(-0.61)$ \\
\hline DIO & & & 0.545 * & $0.553 *$ & & & 0.018 & 0.015 \\
\hline *LowGS & & & $(2.27)$ & $(2.30)$ & & & $(0.68)$ & (0.58) \\
\hline Management & $\begin{array}{c}-2.360+ \\
(-1.85)\end{array}$ & & $\begin{array}{c}-2.169+ \\
(-1.67)\end{array}$ & & $\begin{array}{l}0.032 \\
(0.32)\end{array}$ & & $\begin{array}{l}0.045 \\
(0.44)\end{array}$ & \\
\hline Domestic & $\begin{array}{l}0.215 \\
(090)\end{array}$ & & $\begin{array}{l}0.210 \\
(088)\end{array}$ & & $\begin{array}{l}0.012 \\
(073)\end{array}$ & & $\begin{array}{l}0.012 \\
(076)\end{array}$ & \\
\hline Stable & & $\begin{array}{c}0.671+ \\
(1.85)\end{array}$ & & $\begin{array}{c}0.642+ \\
(1.78)\end{array}$ & & $\begin{array}{c}0.077^{*} \\
(2.23)\end{array}$ & & $\begin{array}{c}0.074 \text { * } \\
(2.17)\end{array}$ \\
\hline
\end{tabular}


Table 4. Cont.

\begin{tabular}{|c|c|c|c|c|c|c|c|c|}
\hline & (1) & (2) & (3) & (4) & (5) & (6) & (7) & (8) \\
\hline & \multicolumn{4}{|c|}{$\mathbf{Q}$} & \multicolumn{4}{|c|}{ ROA } \\
\hline Size & $\begin{array}{l}6.881 \\
(0.22)\end{array}$ & $\begin{array}{l}9.068 \\
(0.29)\end{array}$ & $\begin{array}{l}2.712 \\
(0.09)\end{array}$ & $\begin{array}{l}4.085 \\
(0.13)\end{array}$ & $\begin{array}{c}-7.327^{* *} \\
(-2.80)\end{array}$ & $\begin{array}{c}-7.444^{* *} \\
(-2.81)\end{array}$ & $\begin{array}{c}-7.810 * * \\
(-2.88)\end{array}$ & $\begin{array}{c}-7.979 * * \\
(-2.90)\end{array}$ \\
\hline HighGS & $\begin{array}{l}-9.382 \\
(-1.47)\end{array}$ & $\begin{array}{c}-10.380 \\
(-1.58)\end{array}$ & $\begin{array}{l}-5.960 \\
(-0.90)\end{array}$ & $\begin{array}{l}-6.717 \\
(-0.98)\end{array}$ & $\begin{array}{l}-0.075 \\
(-0.16)\end{array}$ & $\begin{array}{l}-0.065 \\
(-0.14)\end{array}$ & $\begin{array}{l}0.447 \\
(0.93)\end{array}$ & $\begin{array}{l}0.454 \\
(0.94)\end{array}$ \\
\hline LowGS & $\begin{array}{l}-3.020 \\
(-0.60)\end{array}$ & $\begin{array}{l}-1.704 \\
(-0.35)\end{array}$ & $\begin{array}{l}-4.504 \\
(-0.85)\end{array}$ & $\begin{array}{l}-3.569 \\
(-0.68)\end{array}$ & $\begin{array}{l}-0.753 \\
(-1.64)\end{array}$ & $\begin{array}{l}-0.662 \\
(-1.47)\end{array}$ & $\begin{array}{c}-1.004 * \\
(-2.01)\end{array}$ & $\begin{array}{r}-0.937+ \\
(-1.92)\end{array}$ \\
\hline Leverage & $\begin{array}{l}-0.335 \\
(-0.53)\end{array}$ & $\begin{array}{l}-0.290 \\
(-0.46)\end{array}$ & $\begin{array}{l}-0.333 \\
(-0.54)\end{array}$ & $\begin{array}{l}-0.286 \\
(-0.47)\end{array}$ & $\begin{array}{l}0.024 \\
(0.60)\end{array}$ & $\begin{array}{l}0.028 \\
(0.72)\end{array}$ & $\begin{array}{l}0.029 \\
(0.73)\end{array}$ & $\begin{array}{l}0.034 \\
(0.85)\end{array}$ \\
\hline FreeCash & $\begin{array}{c}0.380 * * \\
(3.11)\end{array}$ & $\begin{array}{c}0.387^{* *} \\
(3.11)\end{array}$ & $\begin{array}{c}0.387^{* *} \\
(3.08)\end{array}$ & $\begin{array}{c}0.396^{* *} \\
(3.09)\end{array}$ & $\begin{array}{c}0.088^{* *} \\
(5.00)\end{array}$ & $\begin{array}{c}0.088^{* *} \\
(5.08)\end{array}$ & $\begin{array}{c}0.089^{* *} \\
(5.21)\end{array}$ & $\begin{array}{c}0.089 * * \\
(5.31)\end{array}$ \\
\hline Risk & $\begin{array}{c}7.608+ \\
(1.92)\end{array}$ & $\begin{array}{c}7.011+ \\
(1.72)\end{array}$ & $\begin{array}{c}7.420+ \\
(1.86)\end{array}$ & $\begin{array}{c}6.795+ \\
(1.66)\end{array}$ & $\begin{array}{l}0.146 \\
(0.38)\end{array}$ & $\begin{array}{l}0.054 \\
(0.14)\end{array}$ & $\begin{array}{l}0.074 \\
(0.19)\end{array}$ & $\begin{array}{l}-0.017 \\
(-0.04)\end{array}$ \\
\hline BoardSize & $\begin{array}{l}0.088 \\
(0.17)\end{array}$ & $\begin{array}{l}0.073 \\
(0.13)\end{array}$ & $\begin{array}{l}0.091 \\
(0.17)\end{array}$ & $\begin{array}{l}0.078 \\
(0.14)\end{array}$ & $\begin{array}{l}-0.040 \\
(-1.10)\end{array}$ & $\begin{array}{l}-0.039 \\
(-1.07)\end{array}$ & $\begin{array}{l}-0.040 \\
(-1.12)\end{array}$ & $\begin{array}{l}-0.040 \\
(-1.08)\end{array}$ \\
\hline $\begin{array}{l}\text { Outside } \\
\text { Directors }\end{array}$ & $\begin{array}{l}-0.184 \\
(-1.21)\end{array}$ & $\begin{array}{l}-0.157 \\
(-1.03)\end{array}$ & $\begin{array}{l}-0.211 \\
(-1.40)\end{array}$ & $\begin{array}{l}-0.189 \\
(-1.26)\end{array}$ & $\begin{array}{c}-0.032+ \\
(-1.84)\end{array}$ & $\begin{array}{c}-0.031+ \\
(-1.79)\end{array}$ & $\begin{array}{c}-0.035+ \\
(-1.93)\end{array}$ & $\begin{array}{r}-0.034+ \\
(-1.90)\end{array}$ \\
\hline StockOption & $\begin{array}{l}-0.488 \\
(-0.16)\end{array}$ & $\begin{array}{l}-0.582 \\
(-0.19)\end{array}$ & $\begin{array}{l}-0.753 \\
(-0.24)\end{array}$ & $\begin{array}{l}-0.873 \\
(-0.28)\end{array}$ & $\begin{array}{c}-0.689+ \\
(-1.78)\end{array}$ & $\begin{array}{c}-0.694+ \\
(-1.79)\end{array}$ & $\begin{array}{c}-0.705+ \\
(-1.81)\end{array}$ & $\begin{array}{r}-0.712+ \\
(-1.82)\end{array}$ \\
\hline BigN & $\begin{array}{c}9.883+ \\
(1.83)\end{array}$ & $\begin{array}{c}11.440 * \\
(2.08)\end{array}$ & $\begin{array}{l}9.714 \\
(1.74)\end{array}$ & $\begin{array}{c}11.160+ \\
(1.96)\end{array}$ & $\begin{array}{l}-0.521 \\
(-1.06)\end{array}$ & $\begin{array}{l}-0.462 \\
(-0.91)\end{array}$ & $\begin{array}{l}-0.538 \\
(-1.06)\end{array}$ & $\begin{array}{l}-0.484 \\
(-0.93)\end{array}$ \\
\hline Analyst & $\begin{array}{c}10.790 \text { ** } \\
(3.86)\end{array}$ & $\begin{array}{c}11.460 * * \\
(3.97)\end{array}$ & $\begin{array}{c}10.810^{* *} \\
(3.92)\end{array}$ & $\begin{array}{c}11.460 \text { ** } \\
(4.03)\end{array}$ & $\begin{array}{c}1.101 * * \\
(3.45)\end{array}$ & $\begin{array}{c}1.154^{* *} \\
(3.66)\end{array}$ & $\begin{array}{c}1.084^{* *} \\
(3.37)\end{array}$ & $\begin{array}{c}1.137^{* *} \\
(3.58)\end{array}$ \\
\hline Constant & $\begin{array}{c}-0.22 \\
(-0.00)\end{array}$ & $\begin{array}{l}-59.96 \\
(-0.15)\end{array}$ & $\begin{array}{l}57.59 \\
(0.14)\end{array}$ & $\begin{array}{l}10.55 \\
(0.03)\end{array}$ & $\begin{array}{c}98.43 * * \\
(2.92)\end{array}$ & $\begin{array}{c}96.79 * * \\
(2.89)\end{array}$ & $\begin{array}{c}104.30^{* *} \\
(2.99)\end{array}$ & $\begin{array}{c}103.60 * * \\
(2.98)\end{array}$ \\
\hline Number & 2924 & 2924 & 2924 & 2924 & 2924 & 2924 & 2924 & 2924 \\
\hline AdjustedR2 & 0.120 & 0.118 & 0.124 & 0.123 & 0.206 & 0.211 & 0.208 & 0.213 \\
\hline R2within & 0.126 & 0.124 & 0.132 & 0.130 & 0.212 & 0.217 & 0.215 & 0.220 \\
\hline R2overall & 0.005 & 0.022 & 0.035 & 0.073 & 0.216 & 0.225 & 0.233 & 0.240 \\
\hline R2between & 0.000 & 0.011 & 0.024 & 0.073 & 0.246 & 0.258 & 0.273 & 0.284 \\
\hline$F$ & $9.18^{* *}$ & $9.38^{* *}$ & $7.96^{* *}$ & $8.11^{* *}$ & $11.63^{* *}$ & $12.04^{* *}$ & $10.42^{* *}$ & $10.71 * *$ \\
\hline
\end{tabular}

Note: Observation $=2924$. Standard errors are clustered at the firm level. $t$-values are presented in parentheses. See Table A1 for variable definitions. The results of firm dummies and year dummies are not reported. $+p<0.10$, $* p<0.05,{ }^{* *} p<0.01$.

We further examined whether the other monitoring mechanisms such as audit quality could affect the moderating effects of institutional shareholders. Table 5 presents the results of including the interaction terms of the ownership variables and the dummy of Big $\mathrm{N}$ auditors. In Table 5, we can find that both IO and FO are positive and significant results, consistent with Hypotheses 1A and 1B. As for the interaction terms of $\mathrm{IO}$ (or FO) and the dummy of Big N auditors, the results were not significant. This implies that the other external monitoring mechanisms such as higher audit quality do not give a role of moderating the role of institutional shareholders.

Table 5. Interaction effects of IO and external monitoring on firm performance.

\begin{tabular}{|c|c|c|c|c|c|c|c|c|}
\hline & (1) & (2) & (3) & (4) & (5) & (6) & (7) & (8) \\
\hline & \multicolumn{4}{|c|}{$\mathbf{Q}$} & \multicolumn{4}{|c|}{ ROA } \\
\hline IO & $\begin{array}{c}0.652+ \\
(1.67)\end{array}$ & $\begin{array}{c}0.878^{*} \\
(2.14)\end{array}$ & & & $\begin{array}{c}0.110^{* *} \\
(2.91)\end{array}$ & $\begin{array}{c}0.136^{* *} \\
(3.17)\end{array}$ & & \\
\hline $\begin{array}{c}\mathrm{IO} \\
{ }^{*} \mathrm{BigN}\end{array}$ & $\begin{array}{l}-0.073 \\
(-0.22)\end{array}$ & $\begin{array}{l}-0.088 \\
(-0.26)\end{array}$ & & & $\begin{array}{l}0.014 \\
(0.55)\end{array}$ & $\begin{array}{l}0.013 \\
(0.47)\end{array}$ & & \\
\hline FO & & & $\begin{array}{l}0.839 \\
(1.63)\end{array}$ & $\begin{array}{c}1.073 * \\
(2.01)\end{array}$ & & & $\begin{array}{l}0.124^{*} \\
(2.58)\end{array}$ & $\begin{array}{c}0.149^{* * *} \\
(2.81)\end{array}$ \\
\hline $\mathrm{FO}$ & & & -0.153 & -0.174 & & & 0.034 & 0.032 \\
\hline${ }^{*} \mathrm{BigN}$ & & & $(-0.36)$ & $(-0.40)$ & & & $(1.16)$ & (1.10) \\
\hline
\end{tabular}


Table 5. Cont.

\begin{tabular}{|c|c|c|c|c|c|c|c|c|}
\hline & (1) & (2) & (3) & (4) & (5) & (6) & (7) & (8) \\
\hline & \multicolumn{4}{|c|}{$\mathbf{Q}$} & \multicolumn{4}{|c|}{ ROA } \\
\hline DIO & & & $\begin{array}{l}-0.084 \\
(-0.10)\end{array}$ & $\begin{array}{l}-0.072 \\
(-0.08)\end{array}$ & & & $\begin{array}{c}0.099+ \\
(1.85)\end{array}$ & $\begin{array}{c}0.116^{*} \\
(2.09)\end{array}$ \\
\hline DIO & & & 0.327 & 0.452 & & & -0.009 & -0.009 \\
\hline${ }^{*} \operatorname{BigN}$ & & & $(0.40)$ & $(0.52)$ & & & $(-0.18)$ & $(-0.16)$ \\
\hline Management & $\begin{array}{c}-2.448+ \\
(-1.91)\end{array}$ & & $\begin{array}{c}-2.377+ \\
(-1.86)\end{array}$ & & $\begin{array}{l}0.023 \\
(0.24)\end{array}$ & & $\begin{array}{l}0.029 \\
(0.29)\end{array}$ & \\
\hline Domestic & $\begin{array}{l}0.210 \\
(0.88)\end{array}$ & & $\begin{array}{l}0.196 \\
(0.82)\end{array}$ & & $\begin{array}{l}0.012 \\
(0.70)\end{array}$ & & $\begin{array}{l}0.011 \\
(0.67)\end{array}$ & \\
\hline Stable & & $\begin{array}{c}0.677+ \\
(1.86)\end{array}$ & & $\begin{array}{c}0.619+ \\
(1.70)\end{array}$ & & $\begin{array}{l}0.075 \text { * } \\
(2.17)\end{array}$ & & $\begin{array}{l}0.071 * \\
(2.09)\end{array}$ \\
\hline Size & $\begin{array}{l}7.181 \\
(0.23)\end{array}$ & $\begin{array}{l}9.450 \\
(0.30)\end{array}$ & $\begin{array}{l}5.119 \\
(0.16)\end{array}$ & $\begin{array}{l}6.841 \\
(0.22)\end{array}$ & $\begin{array}{c}-7.306^{* *} \\
(-2.78)\end{array}$ & $\begin{array}{c}-7.413^{* *} \\
(-2.79)\end{array}$ & $\begin{array}{c}-7.666^{* *} \\
(-2.85)\end{array}$ & $\begin{array}{c}-7.814^{* *} \\
(-2.87)\end{array}$ \\
\hline HighGS & $\begin{array}{c}5.454^{*} \\
(1.98)\end{array}$ & $\begin{array}{c}5.149+ \\
(1.91)\end{array}$ & $\begin{array}{l}5.401 \text { * } \\
(2.00)\end{array}$ & $\begin{array}{c}5.106+ \\
(1.93)\end{array}$ & $\begin{array}{c}0.990 * * \\
(3.68)\end{array}$ & $\begin{array}{c}0.984 * * \\
(3.69)\end{array}$ & $\begin{array}{c}0.984^{* *} \\
(3.72)\end{array}$ & $\begin{array}{c}0.980^{* *} \\
(3.73)\end{array}$ \\
\hline LowGS & $\begin{array}{l}4.295 \\
(1.65)\end{array}$ & $\begin{array}{c}4.818+ \\
(1.85)\end{array}$ & $\begin{array}{l}4.312+ \\
(1.66)\end{array}$ & $\begin{array}{c}4.818+ \\
(1.87)\end{array}$ & $\begin{array}{c}-1.076^{* *} \\
(-4.22)\end{array}$ & $\begin{array}{c}-1.081^{* *} \\
(-4.12)\end{array}$ & $\begin{array}{c}-1.043^{* *} \\
(-4.20)\end{array}$ & $\begin{array}{c}-1.047^{* *} \\
(-4.11)\end{array}$ \\
\hline Leverage & $\begin{array}{l}-0.344 \\
(-0.55)\end{array}$ & $\begin{array}{l}-0.295 \\
(-0.47)\end{array}$ & $\begin{array}{l}-0.320 \\
(-0.52)\end{array}$ & $\begin{array}{l}-0.268 \\
(-0.43)\end{array}$ & $\begin{array}{l}0.026 \\
(0.65)\end{array}$ & $\begin{array}{l}0.031 \\
(0.77)\end{array}$ & $\begin{array}{l}0.033 \\
(0.80)\end{array}$ & $\begin{array}{l}0.037 \\
(0.92)\end{array}$ \\
\hline FreeCash & $\begin{array}{c}0.378^{* *} \\
(3.07)\end{array}$ & $\begin{array}{c}0.387^{* *} \\
(3.08)\end{array}$ & $\begin{array}{c}0.383^{* *} \\
(3.09)\end{array}$ & $\begin{array}{c}0.393 \text { ** } \\
(3.11)\end{array}$ & $\begin{array}{c}0.089^{* *} \\
(5.00)\end{array}$ & $\begin{array}{c}0.089^{* *} \\
(5.09)\end{array}$ & $\begin{array}{c}0.089^{* *} \\
(5.16)\end{array}$ & $\begin{array}{c}0.089^{* *} \\
(5.25)\end{array}$ \\
\hline Risk & $\begin{array}{c}7.613+ \\
(1.91)\end{array}$ & $\begin{array}{l}6.909+ \\
(1.68)\end{array}$ & $\begin{array}{c}7.454+ \\
(1.85)\end{array}$ & $\begin{array}{l}6.777 \\
(1.64)\end{array}$ & $\begin{array}{l}0.082 \\
(0.21)\end{array}$ & $\begin{array}{l}-0.015 \\
(-0.04)\end{array}$ & $\begin{array}{l}0.051 \\
(0.13)\end{array}$ & $\begin{array}{l}-0.043 \\
(-0.11)\end{array}$ \\
\hline BoardSize & $\begin{array}{l}0.077 \\
(0.15)\end{array}$ & $\begin{array}{l}0.061 \\
(0.11)\end{array}$ & $\begin{array}{l}0.065 \\
(0.12)\end{array}$ & $\begin{array}{l}0.045 \\
(0.08)\end{array}$ & $\begin{array}{l}-0.042 \\
(-1.16)\end{array}$ & $\begin{array}{l}-0.041 \\
(-1.12)\end{array}$ & $\begin{array}{l}-0.042 \\
(-1.14)\end{array}$ & $\begin{array}{l}-0.041 \\
(-1.10)\end{array}$ \\
\hline $\begin{array}{l}\text { Outside } \\
\text { Directors }\end{array}$ & $\begin{array}{l}-0.188 \\
(-1.22)\end{array}$ & $\begin{array}{l}-0.159 \\
(-1.04)\end{array}$ & $\begin{array}{l}-0.197 \\
(-1.28)\end{array}$ & $\begin{array}{l}-0.172 \\
(-1.12)\end{array}$ & $\begin{array}{c}-0.032+ \\
(-1.82)\end{array}$ & $\begin{array}{c}-0.031+ \\
(-1.76)\end{array}$ & $\begin{array}{c}-0.034+ \\
(-1.92)\end{array}$ & $\begin{array}{c}-0.033+ \\
(-1.88)\end{array}$ \\
\hline StockOption & $\begin{array}{l}-0.548 \\
(-0.18)\end{array}$ & $\begin{array}{l}-0.654 \\
(-0.21)\end{array}$ & $\begin{array}{l}-0.399 \\
(-0.13)\end{array}$ & $\begin{array}{l}-0.471 \\
(-0.15)\end{array}$ & $\begin{array}{c}-0.702+ \\
(-1.81)\end{array}$ & $\begin{array}{c}-0.707+ \\
(-1.82)\end{array}$ & $\begin{array}{c}-0.701+ \\
(-1.79)\end{array}$ & $\begin{array}{c}-0.707+ \\
(-1.80)\end{array}$ \\
\hline BigN & $\begin{array}{l}12.730 \\
(0.98)\end{array}$ & $\begin{array}{l}14.860 \\
(1.08)\end{array}$ & $\begin{array}{l}11.550 \\
(0.89)\end{array}$ & $\begin{array}{l}12.520 \\
(0.94)\end{array}$ & $\begin{array}{l}-0.990 \\
(-0.97)\end{array}$ & $\begin{array}{l}-0.865 \\
(-0.83)\end{array}$ & $\begin{array}{l}-1.249 \\
(-1.22)\end{array}$ & $\begin{array}{l}-1.158 \\
(-1.13)\end{array}$ \\
\hline Analyst & $\begin{array}{c}10.540^{* *} \\
(3.81)\end{array}$ & $\begin{array}{c}11.220 * * \\
(3.92)\end{array}$ & $\begin{array}{c}10.640^{* *} \\
(3.85)\end{array}$ & $\begin{array}{c}11.300 * * \\
(3.95)\end{array}$ & $\begin{array}{c}1.099^{* *} \\
(3.47)\end{array}$ & $\begin{array}{c}1.152^{* *} \\
(3.68)\end{array}$ & $\begin{array}{c}1.084^{* *} \\
(3.40)\end{array}$ & $\begin{array}{c}1.136^{* *} \\
(3.61)\end{array}$ \\
\hline Constant & $\begin{array}{l}-12.08 \\
(-0.03)\end{array}$ & $\begin{array}{l}-73.86 \\
(-0.18)\end{array}$ & $\begin{array}{l}16.83 \\
(0.04)\end{array}$ & $\begin{array}{l}-34.26 \\
(-0.09)\end{array}$ & $\begin{array}{c}98.48^{* *} \\
(2.91)\end{array}$ & $\begin{array}{c}96.76^{* *} \\
(2.88)\end{array}$ & $\begin{array}{c}102.80^{* *} \\
(2.97)\end{array}$ & $\begin{array}{c}101.80 \text { ** } \\
(2.96)\end{array}$ \\
\hline Number & 2924 & 2924 & 2924 & 2924 & 2924 & 2924 & 2924 & 2924 \\
\hline AdjustedR2 & 0.116 & 0.114 & 0.116 & 0.114 & 0.203 & 0.208 & 0.206 & 0.211 \\
\hline R2within & 0.123 & 0.120 & 0.123 & 0.121 & 0.209 & 0.213 & 0.212 & 0.217 \\
\hline R2overall & 0.004 & 0.019 & 0.016 & 0.045 & 0.215 & 0.224 & 0.232 & 0.241 \\
\hline R2between & 0.000 & 0.009 & 0.007 & 0.040 & 0.244 & 0.257 & 0.272 & 0.285 \\
\hline$F$ & $9.39 * *$ & $9.79 * *$ & $8.74^{* *}$ & $8.98 * *$ & $11.63^{* *}$ & $12.09 * *$ & $11.11^{* *}$ & $11.32 * *$ \\
\hline
\end{tabular}

Note: Observations $=2,924$. Standard errors are clustered at the firm level. $t$-values are presented in parentheses. See Table A1 for variable definitions. The results of firm dummies and year dummies are not reported. $+p<0.10$, ${ }^{*} p<0.05,{ }^{* *} p<0.01$.

The results of this subsection are summarized as follows. First, the findings show that institutional shareholders and foreign investors are functioning effectively as monitors, consistent with Hypotheses $1 \mathrm{~A}$ and $1 \mathrm{~B}$. This reflects the weakened role of the main Japanese banks and the possibility that institutional shareholders can serve as substitutes for the banks' prior level of influence. Second, the results are consistent with Hypotheses 2A and 2B. This implies that institutional investors or foreign investors are also interested in investing in and monitoring firms with higher sales growth. These results are interpreted as showing that foreign investors effectively play a monitoring role in Japanese corporations.

\subsection{Robustness}

We confirmed the robustness of our results using two additional analyses First, we adopted the stability of Tobin's $Q$ and ROA as the proxy of sustainable performance. In some cases, 
stakeholder-oriented corporate governance might sacrifice corporate performance, measured as ROA to keep a sustainable relationship with various connected stakeholders. In this case, the stability of corporate performance is an important goal for firms under stakeholder-oriented corporate governance.

Table 6 shows the estimated results to adopt the stability of ROA. Using this table, we find that IO and FO were significant and negative to the stability of ROA. This implies that institutional and foreign shareholders are helpful to stabilize corporate performance. Thus, we interpret that both corporate performance and sustainable performance are enhanced by monitoring the roles of IO and FO.

Table 6. Estimated results of standard deviation of ROA.

\begin{tabular}{|c|c|c|c|c|}
\hline & (1) & (2) & (3) & (4) \\
\hline & \multicolumn{4}{|c|}{ Standard Deviations of ROA } \\
\hline $\mathrm{IO}$ & $\begin{array}{c}-0.048^{* *} \\
(-3.52)\end{array}$ & $\begin{array}{c}-0.058 * \\
(-2.46)\end{array}$ & & \\
\hline $\mathrm{FO}$ & & & $\begin{array}{c}-0.041^{* *} \\
(-2.60)\end{array}$ & $\begin{array}{c}-0.051+ \\
(-1.89)\end{array}$ \\
\hline DIO & & & $\begin{array}{c}-0.056^{* *} \\
(-2.60)\end{array}$ & $\begin{array}{c}-0.094 \text { * } \\
(-2.50)\end{array}$ \\
\hline Management & $\begin{array}{c}0.323+ \\
(1.87)\end{array}$ & & $\begin{array}{c}0.319+ \\
(1.83)\end{array}$ & \\
\hline Domestic & $\begin{array}{c}-0.022 * \\
(-2.30)\end{array}$ & & $\begin{array}{c}-0.017+ \\
(-1.80)\end{array}$ & \\
\hline Stable & & $\begin{array}{c}-0.018+ \\
(-1.78)\end{array}$ & & $\begin{array}{l}-0.017 \\
(-1.65)\end{array}$ \\
\hline Size & $\begin{array}{l}-0.006 \\
(-0.04)\end{array}$ & $\begin{array}{l}-0.127 \\
(-0.85)\end{array}$ & $\begin{array}{l}-0.016 \\
(-0.09)\end{array}$ & $\begin{array}{l}-0.158 \\
(-1.10)\end{array}$ \\
\hline HighGS & $\begin{array}{l}0.956 \\
(1.17)\end{array}$ & $\begin{array}{l}1.262 \\
(1.61)\end{array}$ & $\begin{array}{l}0.925 \\
(1.14)\end{array}$ & $\begin{array}{l}1.145 \\
(1.45)\end{array}$ \\
\hline LowGS & $\begin{array}{c}5.035 * * \\
(3.31)\end{array}$ & $\begin{array}{c}4.648 * \\
(2.30)\end{array}$ & $\begin{array}{c}5.043^{* *} \\
(3.28)\end{array}$ & $\begin{array}{c}4.638^{*} \\
(2.33)\end{array}$ \\
\hline Leverage & $\begin{array}{l}-0.009 \\
(-1.17)\end{array}$ & $\begin{array}{l}-0.010 \\
(-1.26)\end{array}$ & $\begin{array}{l}-0.007 \\
(-0.91)\end{array}$ & $\begin{array}{l}-0.008 \\
(-1.05)\end{array}$ \\
\hline FreeCash & $\begin{array}{c}0.252 * * \\
(2.82)\end{array}$ & $\begin{array}{c}0.346 * \\
(1.99)\end{array}$ & $\begin{array}{c}0.266^{* *} \\
(2.88)\end{array}$ & $\begin{array}{c}0.358 \text { * } \\
(2.01)\end{array}$ \\
\hline Risk & $\begin{array}{c}1.700 * * \\
(4.03)\end{array}$ & $\begin{array}{c}1.625^{* *} \\
(3.51)\end{array}$ & $\begin{array}{c}1.672 * * \\
(4.01)\end{array}$ & $\begin{array}{c}1.683^{* *} \\
(3.48)\end{array}$ \\
\hline BoardSize & $\begin{array}{l}0.003 \\
(0.06)\end{array}$ & $\begin{array}{l}-0.049 \\
(-1.43)\end{array}$ & $\begin{array}{l}0.003 \\
(0.06)\end{array}$ & $\begin{array}{l}-0.045 \\
(-1.36)\end{array}$ \\
\hline $\begin{array}{l}\text { Outside } \\
\text { Directors }\end{array}$ & $\begin{array}{l}-0.009 \\
(-0.86)\end{array}$ & $\begin{array}{c}-0.017+ \\
(-1.74)\end{array}$ & $\begin{array}{l}-0.007 \\
(-0.59)\end{array}$ & $\begin{array}{l}-0.013 \\
(-1.53)\end{array}$ \\
\hline StockOption & $\begin{array}{l}0.074 \\
(0.31)\end{array}$ & $\begin{array}{l}0.306 \\
(0.96)\end{array}$ & $\begin{array}{l}0.069 \\
(0.28)\end{array}$ & $\begin{array}{l}0.316 \\
(0.94)\end{array}$ \\
\hline BigN & $\begin{array}{l}-0.223 \\
(-0.78)\end{array}$ & $\begin{array}{l}-0.298 \\
(-1.02)\end{array}$ & $\begin{array}{l}-0.250 \\
(-0.87)\end{array}$ & $\begin{array}{l}-0.347 \\
(-1.15)\end{array}$ \\
\hline Analyst & $\begin{array}{c}0.652 * * \\
(3.79)\end{array}$ & $\begin{array}{c}0.836^{* *} \\
(3.55)\end{array}$ & $\begin{array}{c}0.637^{* *} \\
(3.77)\end{array}$ & $\begin{array}{c}0.875^{* *} \\
(3.37)\end{array}$ \\
\hline Constant & $\begin{array}{l}-2.294 \\
(-0.89)\end{array}$ & $\begin{array}{l}0.640 \\
(0.33) \\
\end{array}$ & $\begin{array}{l}-2.321 \\
(-0.84)\end{array}$ & $\begin{array}{l}0.980 \\
(0.53)\end{array}$ \\
\hline Number & 433 & 433 & 433 & 433 \\
\hline AdjustedR2 & 0.486 & 0.322 & 0.484 & 0.332 \\
\hline$F$ & $5.96^{* *}$ & $6.42 * *$ & $5.58 * *$ & $5.99 * *$ \\
\hline
\end{tabular}

Following [80], standard deviations are calculated over the sample period. $T$-values are presented in parentheses. See Table A1 for variable definitions. $+p<0.10,{ }^{*} p<0.05,{ }^{* *} p<0.01$.

Second, we focused on the effect of regulation such as the establishment of the Stewardship Code in Table 7. To confirm whether this regulation affects the monitoring role of institutional shareholders, we used a post-period dummy which equals to 1 after the period of the accounting year 2014. We 
interacted post dummy with IO, FO, and DIO to confirm the effect of the Stewardship Code towards the monitoring roles of institutional shareholders. Using this table, we found that IO and FO were positively significant. In addition, we found that the monitoring effect of $\mathrm{IO}$ and $\mathrm{FO}$ has strengthened post the establishment of the Stewardship Code establishment.

Table 7. Effect of Stewardship Code on the results of Tobin's Q and ROA.

\begin{tabular}{|c|c|c|c|c|c|c|c|c|}
\hline & (1) & (2) & (3) & (4) & (5) & (6) & (7) & (8) \\
\hline & \multicolumn{4}{|c|}{$\mathbf{Q}$} & \multicolumn{4}{|c|}{ ROA } \\
\hline $\mathrm{IO}$ & $\begin{array}{l}0.428 \\
(1.54)\end{array}$ & $\begin{array}{l}0.643 * \\
(2.04)\end{array}$ & & & $\begin{array}{c}0.111^{* *} \\
(3.21)\end{array}$ & $\begin{array}{c}0.136^{* *} \\
(3.36)\end{array}$ & & \\
\hline $\begin{array}{c}\text { IO } \\
\text { *Post }\end{array}$ & $\begin{array}{c}0.413^{* *} \\
(2.59)\end{array}$ & $\begin{array}{c}0.442 * * \\
(2.74)\end{array}$ & & & $\begin{array}{c}0.032 * * \\
(3.11)\end{array}$ & $\begin{array}{c}0.033^{* *} \\
(3.26)\end{array}$ & & \\
\hline $\mathrm{FO}$ & & & $\begin{array}{l}0.505 \\
(1.39)\end{array}$ & $\begin{array}{c}0.723+ \\
(1.86)\end{array}$ & & & $\begin{array}{c}0.143 \text { ** } \\
(3.09)\end{array}$ & $\begin{array}{c}0.166^{* *} \\
(3.21)\end{array}$ \\
\hline $\mathrm{FO}$ & & & $0.506^{* *}$ & $0.548^{* *}$ & & & 0.012 & 0.013 \\
\hline${ }^{*}$ Post & & & $(2.97)$ & $(3.13)$ & & & $(0.98)$ & (1.10) \\
\hline DIO & & & $\begin{array}{l}0.269 \\
(0.67)\end{array}$ & $\begin{array}{l}0.426 \\
(1.00)\end{array}$ & & & $\begin{array}{l}0.078 \text { * } \\
(2.30)\end{array}$ & $\begin{array}{l}0.096 * \\
(2.57)\end{array}$ \\
\hline $\begin{array}{l}\text { DIO } \\
\text { *Post }\end{array}$ & & & $\begin{array}{l}-0.118 \\
(-0.39)\end{array}$ & $\begin{array}{l}-0.184 \\
(-0.61)\end{array}$ & & & $\begin{array}{c}0.063 \text { * } \\
(2.30)\end{array}$ & $\begin{array}{l}0.061 \text { * } \\
(2.30)\end{array}$ \\
\hline Management & $\begin{array}{c}-2.353+ \\
(-1.83)\end{array}$ & & $\begin{array}{c}-2.205+ \\
(-1.69)\end{array}$ & & $\begin{array}{l}0.031 \\
(0.31)\end{array}$ & & $\begin{array}{l}0.025 \\
(0.24)\end{array}$ & \\
\hline Domestic & $\begin{array}{l}0.197 \\
(0.84)\end{array}$ & & $\begin{array}{l}0.182 \\
(0.76)\end{array}$ & & $\begin{array}{l}0.011 \\
(0.65)\end{array}$ & & $\begin{array}{l}0.012 \\
(0.70)\end{array}$ & \\
\hline Stable & & $\begin{array}{c}0.706+ \\
(1.93)\end{array}$ & & $\begin{array}{c}0.652+ \\
(1.77)\end{array}$ & & $\begin{array}{c}0.077^{*} \\
(2.23)\end{array}$ & & $\begin{array}{c}0.071 * \\
(2.09)\end{array}$ \\
\hline Size & $\begin{array}{l}5.051 \\
(0.16)\end{array}$ & $\begin{array}{l}6.969 \\
(0.22)\end{array}$ & $\begin{array}{l}1.325 \\
(0.04)\end{array}$ & $\begin{array}{l}2.332 \\
(0.07)\end{array}$ & $\begin{array}{c}-7.476^{* *} \\
(-2.86)\end{array}$ & $\begin{array}{c}-7.605^{* *} \\
(-2.88)\end{array}$ & $\begin{array}{c}-7.653 \text { ** } \\
(-2.87)\end{array}$ & $\begin{array}{c}-7.807^{* *} \\
(-2.88)\end{array}$ \\
\hline HighGS & $\begin{array}{c}5.005+ \\
(1.85)\end{array}$ & $\begin{array}{c}4.680+ \\
(1.77)\end{array}$ & $\begin{array}{c}5.025+ \\
(1.87)\end{array}$ & $\begin{array}{c}4.731+ \\
(1.81)\end{array}$ & $\begin{array}{c}0.957^{* *} \\
(3.55)\end{array}$ & $\begin{array}{c}0.951^{* *} \\
(3.56)\end{array}$ & $\begin{array}{c}0.963^{* *} \\
(3.64)\end{array}$ & $\begin{array}{c}0.958^{* *} \\
(3.64)\end{array}$ \\
\hline LowGS & $\begin{array}{c}4.506+ \\
(1.72)\end{array}$ & $\begin{array}{c}5.019+ \\
(1.93)\end{array}$ & $\begin{array}{c}4.495+ \\
(1.73)\end{array}$ & $\begin{array}{c}4.978+ \\
(1.92)\end{array}$ & $\begin{array}{c}-1.058^{* *} \\
(-4.13)\end{array}$ & $\begin{array}{c}-1.064^{* *} \\
(-4.05)\end{array}$ & $\begin{array}{c}-1.029 * * \\
(-4.14)\end{array}$ & $\begin{array}{c}-1.032 \text { ** } \\
(-4.05)\end{array}$ \\
\hline Leverage & $\begin{array}{l}-0.358 \\
(-0.57)\end{array}$ & $\begin{array}{l}-0.307 \\
(-0.49)\end{array}$ & $\begin{array}{l}-0.333 \\
(-0.54)\end{array}$ & $\begin{array}{l}-0.278 \\
(-0.45)\end{array}$ & $\begin{array}{l}0.025 \\
(0.62)\end{array}$ & $\begin{array}{l}0.030 \\
(0.75)\end{array}$ & $\begin{array}{l}0.031 \\
(0.75)\end{array}$ & $\begin{array}{l}0.035 \\
(0.87)\end{array}$ \\
\hline FreeCash & $\begin{array}{c}0.362 * * \\
(2.99)\end{array}$ & $\begin{array}{c}0.369 * * \\
(3.00)\end{array}$ & $\begin{array}{c}0.366^{* *} \\
(2.99)\end{array}$ & $\begin{array}{c}0.374^{* *} \\
(3.00)\end{array}$ & $\begin{array}{c}0.088^{* *} \\
(4.94)\end{array}$ & $\begin{array}{c}0.088^{* *} \\
(5.02)\end{array}$ & $\begin{array}{c}0.088^{* *} \\
(5.13)\end{array}$ & $\begin{array}{c}0.088^{* *} \\
(5.22)\end{array}$ \\
\hline Risk & $\begin{array}{c}9.806 \text { * } \\
(2.37)\end{array}$ & $\begin{array}{c}9.197 \text { * } \\
(2.15)\end{array}$ & $\begin{array}{l}8.896 * \\
(2.14)\end{array}$ & $\begin{array}{c}8.168+ \\
(1.91)\end{array}$ & $\begin{array}{l}0.248 \\
(0.63)\end{array}$ & $\begin{array}{l}0.153 \\
(0.37)\end{array}$ & $\begin{array}{l}0.198 \\
(0.49)\end{array}$ & $\begin{array}{l}0.107 \\
(0.26)\end{array}$ \\
\hline BoardSize & $\begin{array}{l}0.192 \\
(0.37)\end{array}$ & $\begin{array}{l}0.186 \\
(0.34)\end{array}$ & $\begin{array}{l}0.201 \\
(0.38)\end{array}$ & $\begin{array}{l}0.193 \\
(0.35)\end{array}$ & $\begin{array}{l}-0.032 \\
(-0.88)\end{array}$ & $\begin{array}{l}-0.031 \\
(-0.83)\end{array}$ & $\begin{array}{l}-0.033 \\
(-0.92)\end{array}$ & $\begin{array}{l}-0.033 \\
(-0.87)\end{array}$ \\
\hline $\begin{array}{l}\text { Outside } \\
\text { Directors }\end{array}$ & $\begin{array}{l}-0.198 \\
(-1.30)\end{array}$ & $\begin{array}{l}-0.170 \\
(-1.12)\end{array}$ & $\begin{array}{l}-0.196 \\
(-1.25)\end{array}$ & $\begin{array}{l}-0.172 \\
(-1.10)\end{array}$ & $\begin{array}{c}-0.033+ \\
(-1.87)\end{array}$ & $\begin{array}{c}-0.031+ \\
(-1.82)\end{array}$ & $\begin{array}{c}-0.033+ \\
(-1.86)\end{array}$ & $\begin{array}{c}-0.032+ \\
(-1.81)\end{array}$ \\
\hline StockOption & $\begin{array}{l}-0.609 \\
(-0.20)\end{array}$ & $\begin{array}{l}-0.720 \\
(-0.23)\end{array}$ & $\begin{array}{l}-0.815 \\
(-0.27)\end{array}$ & $\begin{array}{l}-0.944 \\
(-0.30)\end{array}$ & $\begin{array}{c}-0.704+ \\
(-1.83)\end{array}$ & $\begin{array}{c}-0.710+ \\
(-1.84)\end{array}$ & $\begin{array}{c}-0.692+ \\
(-1.77)\end{array}$ & $\begin{array}{c}-0.700+ \\
(-1.79)\end{array}$ \\
\hline BigN & $\begin{array}{c}9.151+ \\
(1.67)\end{array}$ & $\begin{array}{c}10.710+ \\
(1.92)\end{array}$ & $\begin{array}{c}9.800+ \\
(1.69)\end{array}$ & $\begin{array}{c}11.390+ \\
(1.93)\end{array}$ & $\begin{array}{l}-0.585 \\
(-1.14)\end{array}$ & $\begin{array}{l}-0.529 \\
(-1.00)\end{array}$ & $\begin{array}{l}-0.548 \\
(-1.08)\end{array}$ & $\begin{array}{l}-0.488 \\
(-0.93)\end{array}$ \\
\hline Analyst & $\begin{array}{c}11.190 * * \\
(4.00)\end{array}$ & $\begin{array}{c}11.930 \text { ** } \\
(4.10)\end{array}$ & $\begin{array}{c}11.220 * * \\
(4.05)\end{array}$ & $\begin{array}{c}11.900 * * \\
(4.17)\end{array}$ & $\begin{array}{c}1.142 \text { ** } \\
(3.58)\end{array}$ & $\begin{array}{c}1.198^{* *} \\
(3.80)\end{array}$ & $\begin{array}{c}1.122 * * \\
(3.49)\end{array}$ & $\begin{array}{c}1.176^{* *} \\
(3.71)\end{array}$ \\
\hline Constant & $\begin{array}{c}3.74 \\
(0.01)\end{array}$ & $\begin{array}{l}-55.43 \\
(-0.14)\end{array}$ & $\begin{array}{l}56.53 \\
(0.14)\end{array}$ & $\begin{array}{l}12.49 \\
(0.03)\end{array}$ & $\begin{array}{c}99.15^{* *} \\
(2.95)\end{array}$ & $\begin{array}{c}97.61^{* *} \\
(2.92)\end{array}$ & $\begin{array}{c}100.90^{* *} \\
(2.95)\end{array}$ & $\begin{array}{c}100.10^{* *} \\
(2.94)\end{array}$ \\
\hline Number & 2924 & 2924 & 2924 & 2924 & 2924 & 2924 & 2924 & 2924 \\
\hline AdjustedR2 & 0.122 & 0.121 & 0.123 & 0.122 & 0.207 & 0.212 & 0.209 & 0.214 \\
\hline R2within & 0.128 & 0.127 & 0.130 & 0.128 & 0.212 & 0.218 & 0.215 & 0.220 \\
\hline R2overall & 0.010 & 0.032 & 0.038 & 0.083 & 0.214 & 0.223 & 0.230 & 0.239 \\
\hline R2between & 0.001 & 0.020 & 0.027 & 0.085 & 0.243 & 0.256 & 0.269 & 0.282 \\
\hline$F$ & $9.75^{* *}$ & $10.16^{* *}$ & $9.10 * *$ & $9.44^{* *}$ & $11.98^{* *}$ & 12.34 ** & $10.79 * *$ & $11.11^{* *}$ \\
\hline
\end{tabular}

Note: Observations $=2924$. Standard errors are clustered at the firm level. $t$-values are presented in parentheses. See Table A1 for variable definitions. The results of firm dummies and year dummies are not reported. $+p<0.10$, ${ }^{*} p<0.05,{ }^{* *} p<0.01$. 
Finally, we checked the causal relationship between institutional shareholders and performance using two-stage least square models (2SLS) estimation models. We show the results of the first stage in Table 8. We adopt ADR (American Depository Receipts) dummy as an instrument of IO and FO. As foreign shareholders can decrease the cost of holding and trading ADR listed stocks, they favor them. In addition, ADR is an important determinant of institutional shareholders [81]. ADRs are associated with higher foreign ownership in Japan [82]. In addition, corporate performance would not be affected by the selection of ADRs. Thus, we select ADR as an instrument variable of IO and FO. The results of the 2SLS are reported in Table 9. Table 9 shows that IO and FO were significant and positive to both of Tobin's $Q$ and ROA. Therefore, we confirmed the causal effects of performance and institutional or foreign shareholders.

Table 8. Estimated results of the first stage of IV estimation.

\begin{tabular}{|c|c|c|c|c|}
\hline & (1) & (2) & (3) & (4) \\
\hline & IO & IO & FO & FO \\
\hline ADR & $\begin{array}{c}1.206^{* *} \\
(2.91)\end{array}$ & $\begin{array}{l}0.920 * \\
(2.47)\end{array}$ & $\begin{array}{c}0.979 * * \\
(2.82)\end{array}$ & $\begin{array}{c}0.759 * \\
(2.31)\end{array}$ \\
\hline $\mathrm{DIO}$ & & & $\begin{array}{c}-0.342 \text { ** } \\
(-10.37)\end{array}$ & $\begin{array}{c}-0.383 \text { ** } \\
(-12.13)\end{array}$ \\
\hline Management & $\begin{array}{c}-0.156^{*} \\
(-2.15)\end{array}$ & & $\begin{array}{c}-0.189 * \\
(-2.37)\end{array}$ & \\
\hline Domestic & $\begin{array}{c}-0.165^{* *} \\
(-6.63)\end{array}$ & & $\begin{array}{c}-0.117^{* *} \\
(-5.74)\end{array}$ & \\
\hline Stable & & $\begin{array}{l}-0.461^{* *} \\
(-13.20)\end{array}$ & & $\begin{array}{c}-0.334 \text { ** } \\
(-10.38)\end{array}$ \\
\hline Size & $\begin{array}{l}2.365 \\
(1.75)\end{array}$ & $\begin{array}{c}2.473 * \\
(2.11)\end{array}$ & $\begin{array}{c}4.691 * * \\
(3.94)\end{array}$ & $\begin{array}{c}4.780 * * \\
(4.13)\end{array}$ \\
\hline HighGS & $\begin{array}{l}0.350 \\
(1.63)\end{array}$ & $\begin{array}{l}0.359 \\
(1.78)\end{array}$ & $\begin{array}{l}0.341 \\
(1.83)\end{array}$ & $\begin{array}{l}0.339 \\
(1.91)\end{array}$ \\
\hline LowGS & $\begin{array}{l}-0.403 \\
(-1.71)\end{array}$ & $\begin{array}{l}-0.327 \\
(-1.51)\end{array}$ & $\begin{array}{c}-0.474 \text { * } \\
(-2.37)\end{array}$ & $\begin{array}{c}-0.406 * \\
(-2.14)\end{array}$ \\
\hline Leverage & $\begin{array}{c}-0.120^{* *} \\
(-4.36)\end{array}$ & $\begin{array}{c}-0.127^{* *} \\
(-5.37)\end{array}$ & $\begin{array}{c}-0.136^{* *} \\
(-5.51)\end{array}$ & $\begin{array}{c}-0.141 \text { ** } \\
(-6.09)\end{array}$ \\
\hline FreeCash & $\begin{array}{c}0.062 * * \\
(3.81)\end{array}$ & $\begin{array}{c}0.055^{* *} \\
(3.50)\end{array}$ & $\begin{array}{c}0.045^{* *} \\
(2.77)\end{array}$ & $\begin{array}{c}0.041 * \\
(2.52)\end{array}$ \\
\hline Risk & $\begin{array}{c}1.100^{* *} \\
(2.87)\end{array}$ & $\begin{array}{c}1.469^{* *} \\
(3.88)\end{array}$ & $\begin{array}{c}1.086^{* *} \\
(3.34)\end{array}$ & $\begin{array}{c}1.361^{* *} \\
(4.26)\end{array}$ \\
\hline BoardSize & $\begin{array}{l}0.046 \\
(0.74)\end{array}$ & $\begin{array}{l}0.039 \\
(0.67)\end{array}$ & $\begin{array}{l}0.033 \\
(0.67)\end{array}$ & $\begin{array}{l}0.029 \\
(0.60)\end{array}$ \\
\hline $\begin{array}{l}\text { Outside } \\
\text { Director }\end{array}$ & $\begin{array}{l}0.014 \\
(0.99)\end{array}$ & $\begin{array}{l}0.004 \\
(0.29)\end{array}$ & $\begin{array}{c}0.027 * \\
(2.07)\end{array}$ & $\begin{array}{l}0.020 \\
(1.57)\end{array}$ \\
\hline StockOption & $\begin{array}{c}-0.684 * \\
(-2.00)\end{array}$ & $\begin{array}{l}-0.554 \\
(-1.76)\end{array}$ & $\begin{array}{c}-0.637^{*} \\
(-2.25)\end{array}$ & $\begin{array}{c}-0.542 * \\
(-2.04)\end{array}$ \\
\hline BigN & $\begin{array}{l}1.698 \\
(1.90)\end{array}$ & $\begin{array}{l}1.195 \\
(1.43)\end{array}$ & $\begin{array}{l}0.717 \\
(0.90)\end{array}$ & $\begin{array}{l}0.451 \\
(0.59)\end{array}$ \\
\hline Analyst & $\begin{array}{c}1.418^{* *} \\
(3.47)\end{array}$ & $\begin{array}{c}0.888^{*} \\
(2.34)\end{array}$ & $\begin{array}{c}1.116^{* *} \\
(3.26)\end{array}$ & $\begin{array}{c}0.758^{*} \\
(2.37)\end{array}$ \\
\hline$F$ & $33.16^{* *}$ & $48.29^{* *}$ & $74.69 * *$ & $89.37^{* *}$ \\
\hline
\end{tabular}

Note: $\mathrm{t}$-values are presented in parentheses. See Table A1 for variable definitions. The results of firm dummies and year dummies are not reported. ${ }^{*} p<0.05,{ }^{* *} p<0.01$.

We additionally discuss the robustness of our results in accordance with anonymous referees' comments. First, we also performed an analysis of listed firms on the 1st Section of TSE in the additional un-tabled results. Second, we additionally confirm the robustness of the results from 2007 to 2016. Third, we drop the observations which indicate upper and lower 1\% of Tobin's Q and ROA in our samples. Using these un-tabulated results, we also confirm similar results. 
Table 9. Estimated results of Tobin's Q and ROA (IV estimation).

\begin{tabular}{|c|c|c|c|c|c|c|c|c|}
\hline & (1) & (2) & (3) & (4) & (5) & (6) & (7) & (8) \\
\hline & \multicolumn{4}{|c|}{$\mathbf{Q}$} & \multicolumn{4}{|c|}{ ROA } \\
\hline $\mathrm{IO}$ & $\begin{array}{c}11.320^{* *} \\
(2.69)\end{array}$ & $\begin{array}{c}14.790 * \\
(2.36)\end{array}$ & & & $\begin{array}{l}0.816^{*} \\
(2.28)\end{array}$ & $\begin{array}{c}1.057^{*} \\
(2.05)\end{array}$ & & \\
\hline FO & & & $\begin{array}{c}14.000 * * \\
(2.63)\end{array}$ & $\begin{array}{c}18.000 * \\
(2.23)\end{array}$ & & & $\begin{array}{l}1.007^{*} \\
(2.27)\end{array}$ & $\begin{array}{c}1.283 * \\
(1.99)\end{array}$ \\
\hline DIO & & & $\begin{array}{c}5.065^{* *} \\
(2.68)\end{array}$ & $\begin{array}{c}7.167^{*} \\
(2.26)\end{array}$ & & & $\begin{array}{c}0.356 \text { * } \\
(2.30)\end{array}$ & $\begin{array}{c}0.502 * \\
(2.01)\end{array}$ \\
\hline Management & $\begin{array}{l}-0.486 \\
(-0.44)\end{array}$ & & $\begin{array}{l}0.378 \\
(0.25)\end{array}$ & & $\begin{array}{l}0.132 \\
(1.33)\end{array}$ & & $\begin{array}{l}0.194 \\
(1.49)\end{array}$ & \\
\hline Domestic & $\begin{array}{c}2.007^{* *} \\
(2.71)\end{array}$ & & $\begin{array}{c}1.795^{* *} \\
(2.67)\end{array}$ & & $\begin{array}{c}0.115+ \\
(1.85)\end{array}$ & & $\begin{array}{c}0.100+ \\
(1.79)\end{array}$ & \\
\hline Stable & & $\begin{array}{c}6.972 * \\
(2.37)\end{array}$ & & $\begin{array}{c}6.191 \text { * } \\
(2.27)\end{array}$ & & $\begin{array}{c}0.465+ \\
(1.93)\end{array}$ & & $\begin{array}{c}0.407+ \\
(1.87)\end{array}$ \\
\hline Size & $\begin{array}{l}-4.688 \\
(-0.20)\end{array}$ & $\begin{array}{c}-11.720 \\
(-0.43)\end{array}$ & $\begin{array}{c}-43.110 \\
(-1.29)\end{array}$ & $\begin{array}{c}-60.680 \\
(-1.31)\end{array}$ & $\begin{array}{l}1.486 \\
(0.73)\end{array}$ & $\begin{array}{l}0.763 \\
(0.34)\end{array}$ & $\begin{array}{l}-1.289 \\
(-0.47)\end{array}$ & $\begin{array}{l}-2.738 \\
(-0.75)\end{array}$ \\
\hline HighGS & $\begin{array}{l}4.705 \\
(1.62)\end{array}$ & $\begin{array}{l}3.130 \\
(0.87)\end{array}$ & $\begin{array}{l}3.912 \\
(1.21)\end{array}$ & $\begin{array}{l}2.355 \\
(0.58)\end{array}$ & $\begin{array}{c}1.207^{* *} \\
(4.79)\end{array}$ & $\begin{array}{c}1.119^{* *} \\
(3.70)\end{array}$ & $\begin{array}{c}1.150^{* *} \\
(4.26)\end{array}$ & $\begin{array}{c}1.064^{* *} \\
(3.25)\end{array}$ \\
\hline LowGS & $\begin{array}{l}3.188 \\
(0.95)\end{array}$ & $\begin{array}{l}3.929 \\
(1.01)\end{array}$ & $\begin{array}{l}5.278 \\
(1.39)\end{array}$ & $\begin{array}{l}6.416 \\
(1.36)\end{array}$ & $\begin{array}{c}-1.884^{* *} \\
(-6.28)\end{array}$ & $\begin{array}{c}-1.869 * * \\
(-5.61)\end{array}$ & $\begin{array}{c}-1.735^{* *} \\
(-5.23)\end{array}$ & $\begin{array}{c}-1.692 * * \\
(-4.36)\end{array}$ \\
\hline Leverage & $\begin{array}{l}0.818 \\
(1.20)\end{array}$ & $\begin{array}{l}1.356 \\
(1.44)\end{array}$ & $\begin{array}{l}1.367 \\
(1.61)\end{array}$ & $\begin{array}{l}2.015 \\
(1.59)\end{array}$ & $\begin{array}{l}0.006 \\
(0.10)\end{array}$ & $\begin{array}{l}0.044 \\
(0.53)\end{array}$ & $\begin{array}{l}0.046 \\
(0.60)\end{array}$ & $\begin{array}{l}0.090 \\
(0.86)\end{array}$ \\
\hline FreeCash & $\begin{array}{l}0.093 \\
(0.29)\end{array}$ & $\begin{array}{l}0.002 \\
(0.00)\end{array}$ & $\begin{array}{l}0.160 \\
(0.49)\end{array}$ & $\begin{array}{l}0.076 \\
(0.18)\end{array}$ & $\begin{array}{c}0.151^{* *} \\
(2.81)\end{array}$ & $\begin{array}{c}0.144^{* *} \\
(2.62)\end{array}$ & $\begin{array}{c}0.156^{* *} \\
(3.00)\end{array}$ & $\begin{array}{c}0.149 * * \\
(2.80)\end{array}$ \\
\hline Risk & $\begin{array}{l}-2.530 \\
(-0.39)\end{array}$ & $\begin{array}{c}-11.790 \\
(-1.08)\end{array}$ & $\begin{array}{l}-5.320 \\
(-0.72)\end{array}$ & $\begin{array}{c}-14.600 \\
(-1.17)\end{array}$ & $\begin{array}{l}-0.480 \\
(-0.88)\end{array}$ & $\begin{array}{l}-1.127 \\
(-1.29)\end{array}$ & $\begin{array}{l}-0.678 \\
(-1.13)\end{array}$ & $\begin{array}{l}-1.322 \\
(-1.36)\end{array}$ \\
\hline BoardSize & $\begin{array}{l}-0.802 \\
(-1.06)\end{array}$ & $\begin{array}{l}-0.881 \\
(-0.96)\end{array}$ & $\begin{array}{l}-0.757 \\
(-0.98)\end{array}$ & $\begin{array}{l}-0.834 \\
(-0.87)\end{array}$ & $\begin{array}{l}-0.074 \\
(-1.14)\end{array}$ & $\begin{array}{l}-0.077 \\
(-1.04)\end{array}$ & $\begin{array}{l}-0.070 \\
(-1.12)\end{array}$ & $\begin{array}{l}-0.074 \\
(-1.00)\end{array}$ \\
\hline $\begin{array}{l}\text { Outside } \\
\text { Director }\end{array}$ & $\begin{array}{c}-0.418^{*} \\
(-2.15)\end{array}$ & $\begin{array}{l}-0.297 \\
(-1.41)\end{array}$ & $\begin{array}{c}-0.634 \text { * } \\
(-2.54)\end{array}$ & $\begin{array}{c}-0.587^{*} \\
(-2.04)\end{array}$ & $\begin{array}{l}-0.022 \\
(-1.15)\end{array}$ & $\begin{array}{l}-0.014 \\
(-0.75)\end{array}$ & $\begin{array}{c}-0.037+ \\
(-1.69)\end{array}$ & $\begin{array}{l}-0.035 \\
(-1.49)\end{array}$ \\
\hline StockOption & $\begin{array}{l}5.828 \\
(1.16)\end{array}$ & $\begin{array}{l}6.182 \\
(1.04)\end{array}$ & $\begin{array}{l}6.986 \\
(1.31)\end{array}$ & $\begin{array}{l}7.723 \\
(1.16)\end{array}$ & $\begin{array}{l}-0.148 \\
(-0.34)\end{array}$ & $\begin{array}{l}-0.116 \\
(-0.23)\end{array}$ & $\begin{array}{l}-0.065 \\
(-0.14)\end{array}$ & $\begin{array}{l}-0.006 \\
(-0.01)\end{array}$ \\
\hline BigN & $\begin{array}{l}-7.877 \\
(-0.63)\end{array}$ & $\begin{array}{l}-5.228 \\
(-0.37)\end{array}$ & $\begin{array}{l}0.884 \\
(0.08)\end{array}$ & $\begin{array}{l}3.952 \\
(0.28)\end{array}$ & $\begin{array}{c}-1.741+ \\
(-1.67)\end{array}$ & $\begin{array}{l}-1.629 \\
(-1.41)\end{array}$ & $\begin{array}{l}-1.096 \\
(-1.15)\end{array}$ & $\begin{array}{l}-0.959 \\
(-0.87)\end{array}$ \\
\hline Analyst & $\begin{array}{l}-1.701 \\
(-0.23) \\
\end{array}$ & $\begin{array}{l}1.552 \\
(0.19)\end{array}$ & $\begin{array}{l}-1.405 \\
(-0.19)\end{array}$ & $\begin{array}{l}0.975 \\
(0.12)\end{array}$ & $\begin{array}{l}0.348 \\
(0.57)\end{array}$ & $\begin{array}{l}0.541 \\
(0.89) \\
\end{array}$ & $\begin{array}{l}0.375 \\
(0.64)\end{array}$ & $\begin{array}{l}0.505 \\
(0.81)\end{array}$ \\
\hline$F$ & $6.43^{* *}$ & $5.01 * *$ & $5.78^{* *}$ & $4.47^{* *}$ & $17.57^{* *}$ & 15.22 ** & $16.10^{* *}$ & $13.56^{* *}$ \\
\hline $\begin{array}{c}\text { Under } \\
\text { identification }\end{array}$ & $8.42 * *$ & $6.11 * *$ & $7.95^{* *}$ & $5.36 *$ & $8.42 * *$ & $6.11 *$ & $7.95^{* *}$ & $5.36^{*}$ \\
\hline
\end{tabular}

Note: Observations $=2,924$. $\mathrm{t}$-values are presented in parentheses. See Table A1 for variable definitions. Under identification mean LM test statistic for under-identification (Under-identification test). Instrumented variables are IO in Model (1), (2), (5), (6), and FO in Model (3), (4), (7), and (8). Instrument variable is ADR dummy. The results of firm dummies and year dummies are not reported. $+p<0.10, * p<0.05,{ }^{* *} p<0.01$.

\section{Discussion}

This study analyzed the effect of institutional shareholders on firm performance. Institutional shareholders were divided into foreign and domestic institutional shareholders and the results suggest that these effectively function as monitors in stakeholder-oriented systems. In addition, institutional shareholders, including foreign shareholders, are more effective in monitoring firms with higher growth opportunities. Thus, institutional shareholders contribute to improving firm performance, especially for firms with higher growth opportunities.

This study makes several theoretical contributions. First, it investigates whether institutional shareholders with shareholder-oriented scope effectively perform a monitoring role in stakeholderoriented corporate governance countries such as Japan. The results imply that institutional shareholders are capable of taking on a role that is complementary to bank monitoring in stakeholder-oriented corporate governance environments. Second, this study also reveals that institutional shareholders play a stronger monitoring role in firms with higher growth opportunities. This implies that institutional 
shareholders or foreign shareholders more effectively monitor firms with higher growth projects, which leads to higher future profitability. These findings suggest that foreign shareholders function effectively as monitors in this transitional era, marking the introduction of a shareholder-oriented system.

There are several limitations found in this study. First, subsequent corporate governance reforms like Japan's Stewardship Code and Japan's Corporate Governance Code have been implemented since 2015. While the dialogue among shareholders based on these two codes is only in its early stages, the effects of the two codes are expected to change the attitudes of institutional shareholders in Japan. Second, the Corporate Governance Code recommends appointing at least two independent directors from 2016 onwards. These subsequent changes for Japanese corporations might function as a mechanism for convergence into the Anglo-American corporate governance system. From the substitute theory perspective [83], board monitoring would matter as a substitute for bank monitoring. Thus, an increase in independent directors might also serve as a substitute for traditional main bank monitoring in Japan. These recent changes should be treated as part of the changing institutional context of stakeholder-oriented corporate governance, and thus provide potential avenues for future research.

\section{Conclusions}

This study investigated the role of institutional investors in Japan following the financial restructuring, which affected the country's main banks. Under a stakeholder-oriented system, previous studies indicated that stable shareholders such as banks, Keiretsu groups, and parent-subsidiary relationships have functioned as effective monitors in Japan. On the other hand, several studies investigated the relationship between foreign ownership and firm performance [26,27]. However, these studies did not focus on either the role of institutional shareholders or the difference between foreign and domestic institutional shareholders. This study focused on the effect of the monitoring role of institutional investors. Thus, the analyses sought to reveal certain aspects of the role of institutional investors, including the difference between foreign and domestic institutional shareholders in stakeholder-oriented corporate governance environments such as Japan.

The results of this study are summarized in the following three points. First, the results show that institutional and foreign shareholders are as effective as monitors in Japanese corporations. In addition, domestic shareholders, including stable shareholders, are also expected to have a monitoring role. This implies that the increased influence of institutional investors functions as a monitoring mechanism that is complementary to that of domestic and stable shareholders. Second, monitoring by institutional and foreign shareholders is expected to strengthen firms with higher growth opportunities.

Our study explored the sustainability of economic growth based on constructive dialogue between a corporation and its institutional investors. Under FSA guidelines, institutional shareholders are expected to engage in proactive dialogue. Therefore, they are able to play an effective and complementary monitoring role in stakeholder-oriented economies, such as Japan. In this sense, the study contributes to the understanding of the roles of institutional investors under non-Anglo-American economies, which differ from shareholder-oriented economies. There might be an empirical question as to whether institutional shareholders effectively function in emerging economies, which do not completely follow a pure market discipline. These might be valuable areas for future research.

Author Contributions: Funding acquisition, H.S. and N.W.; Writing-original draft and editing, H.S. and N.W. All authors have read and agree to the published version of the manuscript.

Funding: Japan Ministry of Education, Culture, Sports, Science and Technology, the Grants-in-Aid for Young Scientists (A) (MEXT/JSPS KAKENHI Grant Number 17H04784); the Grant-in-Aid for Scientific Research (B) (MEXT/JSPS KAKENHI Grant Number 17KT0036); the Grant-in-Aid for Scientific Research (C) (MEXT/JSPS KAKENHI Grant Number 17K03695); Murata Science Foundation; Shikishima foundation (34th Grant-in-Aid for Scientific Research).

Acknowledgments: The authors would like to acknowledge Derek Lehmberg and participants of 32nd annual meeting of the Association of Japanese Business Studies. We would also like to acknowledge Japan Ministry of Education, Culture, Sports, Science and Technology, Murata Science Foundation, and Shikishima foundation for financial assistance. 
Conflicts of Interest: The authors declare no conflict of interest.

\section{Appendix A}

Table A1. Definition of Variables.

\begin{tabular}{|c|c|}
\hline Variable & Definition \\
\hline & Dependent Variables \\
\hline Q & $\begin{array}{l}\text { Tobin's Q: market value of equity and book value of liabilities divided by book value } \\
\text { of assets }\end{array}$ \\
\hline ROA & $\begin{array}{c}\text { Return on assets: current income divided by book value of assets } \\
\text { Ownership Variables }\end{array}$ \\
\hline $\mathrm{IO}$ & $\begin{array}{l}\text { Percentage of institutional shareholdings: institutional investors consist of foreign } \\
\text { investors (excluding foreign corporate investors), trust accounts, and special accounts } \\
\text { of domestic insurance companies [69] }\end{array}$ \\
\hline $\mathrm{FO}$ & Percentage of foreign shareholdings \\
\hline $\mathrm{DIO}$ & $\begin{array}{c}\text { Percentage of domestic institutional shareholdings computed by max }(\mathrm{IO}-\text { percentage } \\
\text { ownership by foreigners, } 0)[70] .\end{array}$ \\
\hline Management & Percentage of shareholdings of the board of directors [51] \\
\hline Domestic & $\begin{array}{l}\text { Total ownership of domestic shareholders such as main banks, and cross and } \\
\text { dominant shareholdings; percentage of main bank shareholdings [51]; Percentage of } \\
\text { cross-shareholdings with other publicly-held companies that are permitted to hold } \\
\text { their shares is calculated by the NLI Research Institute; the percentage of } \\
\text { shareholdings by controlling companies implies that their ownership exceeds } 15 \% \text { of } \\
\text { the outstanding shares }\end{array}$ \\
\hline Stable & $\begin{array}{l}\text { Cumulative percentage of stock held by domestic shareholders such as } \\
\text { cross-shareholders, insurance companies (life/non-life), banks, shinkin banks, } \\
\text { publicly-held companies, publicly-held affiliates, director and employee ownership, } \\
\text { treasury stock, and major corporate shareholders (more than 3\%, including foreign } \\
\text { companies and excluding trust banks) } \\
\text { Other Variables }\end{array}$ \\
\hline Size & The logarithm of firm assets \\
\hline High SG & Equals 1 if firm sales growth is above the 75th percentile; otherwise 0 \\
\hline Low SG & Equals 1 if firm sales growth is below the 25th percentile; otherwise 0 \\
\hline Leverage & Financial leverage; Debt/Total Assets \\
\hline Free Cash & Free Cash Flow/Total Assets \\
\hline Risk & Stock price volatility over three years \\
\hline Board Size & Number of directors on the board \\
\hline Outside Directors & Outside directors/Board size (\%) \\
\hline Stock Option & Stock Option is equal to one if a firm adopts stock options, and zero otherwise. \\
\hline Big N & Big-N audit firms, consisting of Big- 3 or Big- 4 audit firms \\
\hline Analyst & The logarithm of the number of analyst coverage \\
\hline
\end{tabular}

\section{Appendix B}

Table A2. Major corporate governance changes in Japan.

\begin{tabular}{cccc}
\hline Date & Corporate Governance Rule & Subject to Rule & Type of Rule \\
\hline 26 February 2014 & Establishment of Stewardship Code & Institutional Shareholders & Comply or Explain \\
1 July 2015 & Corporate Governance Code & Listed Firms & Comply or Explain \\
29 May 2017 & Revised Version of Stewardship Code & Institutional Shareholders & Comply or Explain \\
\hline
\end{tabular}

Note. This is a summary of the recent changes in CG rules in Japan based on Prime Minister Abe's administration, "Japan is Back.".

\section{References}

1. Aggarwal, R.; Erel, I.; Ferreira, M.; Matos, P. Does governance travel around the world? Evidence from institutional investors. J. Financial Econ. 2011, 100, 154-181. [CrossRef] 
2. Hartzell, J.C.; Starks, L.T. Institutional Investors and Executive Compensation. J. Finance 2003, 58, $2351-2374$. [CrossRef]

3. Pound, J. Proxy contests and the efficiency of shareholder oversight. J. Financial Econ. 1988, 20, $237-265$. [CrossRef]

4. McConnell, J.J.; Servaes, H. Additional evidence on equity ownership and corporate value. J. Financial Econ. 1990, 27, 595-612. [CrossRef]

5. Gillan, S.L.; Starks, L.T. Corporate Governance, Corporate Ownership, and the Role of Institutional Investors: A Global Perspective. SSRN Electron. J. 2003, 13, 4-22. [CrossRef]

6. Yoshimori, M. Whose company is it? The concept of the corporation in Japan and the west. Long Range Plan. 1995, 28, 2-44. [CrossRef]

7. Seki, T. Legal Reform and Shareholder Activism by Institutional Investors in Japan. Corp. Gov. Int. Rev. 2005, 13, 377-385. [CrossRef]

8. Colpan, A.M.; Yoshikawa, T.; Hikino, T.; Miyoshi, H. Japanese Corporate Governance: Structural Change and Financial Performance. Asian Bus. Manag. 2007, 6, S89-S113. [CrossRef]

9. Suto, M.; Toshino, M. Behavioral biases of Japanese institutional investors: Fund management and corporate governance. Corp. Gov.: An Int. Rev. 2005, 13, 466-477. [CrossRef]

10. Investor rights in Japan are overdue for a boost. Financial Times, 2 December 2007.

11. Jensen, M.C.; Meckling, W.H. Theory of the firm: Managerial behavior, agency costs and ownership structure. J. Financial Econ. 1976, 3, 305-360. [CrossRef]

12. Myers, S.C.; Majluf, N.S. Corporate financing and investment decisions when firms have information that investors do not have. J. Financial Econ. 1984, 13, 187-221. [CrossRef]

13. Gine, M.; Moussawi, R.; Sedunov, J. Governance mechanisms and effective activism: Evidence from shareholder proposals on poison pills. J. Empir. Finance 2017, 43, 185-202. [CrossRef]

14. Bushee, B. The influence of institutional investors on myopic R\&D investment behavior. Account. Rev. 1998, $73,19-45$.

15. Bushee, B. Do institutional investors prefers near-term earnings over long-run value? Contemp. Account. Res. 2001, 18, 207-246. [CrossRef]

16. Edmans, A. Blockholder Trading, Market Efficiency, and Managerial Myopia. J. Finance 2009, 64, $2481-2513$. [CrossRef]

17. Desender, K.; Aguilera, R.V.; López-Puertas Lamy, M.; Crespí, R. A clash of governance logics: Foreign ownership and board monitoring. Strateg. Manag. J. 2016, 37, 349-369. [CrossRef]

18. Hoshi, T.; Kashyap, A.; Scharfstein, D. Corporate Structure, Liquidity, and Investment: Evidence from Japanese Industrial Groups. Q. J. Econ. 1991, 106, 33-60. [CrossRef]

19. Aoki, M.; Patrick, H. The Japanese Main Bank System: Its Relevance for Developing and Transforming Economies; Oxford University Press: Oxford, UK, 1994.

20. Hoshi, T.; Kashyap, A.K. Will the U.S. bank recapitalization succeed? Eight lessons from Japan. J. Financial Econ. 2010, 97, 398-417. [CrossRef]

21. An accountability gap is holding back Japan's economy. Financial Times, 14 March 2007.

22. My 'third arrow' will fell Japan's economic demons. Financial Times, 29 June 2014.

23. Miyajima, H.; Hoda, T. Understanding the Changing Ownership Structure of Japanese Firms: Investment Style of Foreign and Domestic Institutional Investors; Discussion Papers, DP2011-11; Japanese Financial Services Agency: Tokyo, Japan, 2012. (in Japanese)

24. Buchanan, J.; Chai, D.H.; Deakin, S. Agency Theory in Practice: A Qualitative Study of Hedge Fund Activism in Japan. Corp. Gov. Int. Rev. 2014, 22, 296-311. [CrossRef]

25. Yoshikawa, T.; Rasheed, A.A.; Del Brio, E.B. The impact of firm strategy and foreign ownership on executive bonus compensation in Japanese firms. J. Bus. Res. 2010, 63, 1254-1260. [CrossRef]

26. Ahmadjian, C.L.; Robbins, G.E. A Clash of Capitalisms: Foreign Shareholders and Corporate Restructuring in 1990s Japan. Am. Sociol. Rev. 2005, 70, 451-471. [CrossRef]

27. David, P.; Yoshikawa, T.; Chari, M.D.R.; Rasheed, A.A. Strategic investments in Japanese corporations: Do foreign portfolio owners foster underinvestment or appropriate investment? Strat. Manag. J. 2006, 27, 591-600. [CrossRef]

28. Sakawa, H.; Watanabel, N. Family control and ownership monitoring in Stakeholder-oriented corporate governance. Manag. Decis. 2019, 57, 1712-1728. [CrossRef] 
29. Sakawa, H.; Ubukata, M.; Watanabel, N. Market liquidity and bank-dominated corporate governance: Evidence from Japan. Int. Rev. Econ. Finance 2014, 31, 1-11. [CrossRef]

30. Yeh, T.-M. Large Shareholders, Shareholder Proposals, and Firm Performance: Evidence from Japan. Corp. Gov. Int. Rev. 2014, 22, 312-329. [CrossRef]

31. Jensen, M.C. Agency costs of free cash flow, corporate finance, and takeovers. Am. Econ. Rev. 1986, 76, 323-329.

32. Aguilera, R.V.; Filatotchev, I.; Gospel, H.; Jackson, G. An Organizational Approach to Comparative Corporate Governance: Costs, Contingencies, and Complementarities. Organ. Sci. 2008, 19, 475-492. [CrossRef]

33. Hall, P.A.; Soskice, D. An Introduction to Varieties of Capitalism; Oxford University Press (OUP): Oxford, UK, 2001; pp. 1-68.

34. Shleifer, A.; Vishny, R.W. A survey of corporate governance. J. Financ. 1997, 52, 737-783. [CrossRef]

35. Dore, R. Stock Market Capitalism: Welfare Capitalism: Japan and Germany Versus the Anglo-Saxons; Oxford University Press: Oxford, UK, 2000.

36. Aguilera, R.V.; Jackson, G. The cross-national diversity of corporate governance: Dimensions and determinants. Acad. Manag. Rev. 2003, 28, 447-465. [CrossRef]

37. Lincoln, J.R.; Gerlach, M.L. Japan's Network Economy: Structure, Persistence, and Change; Cambridge University Press: New York, NY, USA, 2004.

38. Aoki, M. Toward an economic model of the Japanese firm. J. Econ. Lit. 1990, 28, 1-27.

39. Sakawa, H.; Watanabel, N. Main bank relationship and accounting conservatism: Evidence from Japan. Asian Bus. Manag. 2019, 1-24. [CrossRef]

40. Sakawa, H.; Watanabel, N. Earnings quality and internal control in bank-dominated corporate governance. Asian Bus. Manag. 2019, 1-33. [CrossRef]

41. Sakawa, H.; Watanabel, N. IPO underpricing and ownership monitoring in Japan. Asian Bus. Manag. 2019, 1-24. [CrossRef]

42. Hansmann, H.; Kraakman, R. The end of history for corporate law. Georgetown Law J. 2001, 89, 439-468.

43. Khanna, T.; Palepu, K.G. Globalization and convergence in corporate governance: Evidence from Infosys and the Indian software industry. J. Int. Bus. Stud. 2004, 35, 484-507. [CrossRef]

44. Useem, M. Corporate Leadership in a Globalizing Equity Market. Acad. Manag. Perspect. 1998, 12, 43-59. [CrossRef]

45. Chizema, A.; Shinozawa, Y. The 'company with committees': Change or continuity in Japanese corporate governance? J. Manag. Stud. 2012, 49,77-101. [CrossRef]

46. Sakawa, H.; Moriyama, K.; Watanabel, N. Relation between Top Executive Compensation Structure and Corporate Governance: Evidence from Japanese Public Disclosed Data. Corp. Gov. Int. Rev. 2012, 20, 593-608. [CrossRef]

47. Geng, X.; Yoshikawa, T.; Colpan, A.M. Leveraging foreign institutional logic in the adoption of stock option pay among Japanese firms. Strateg. Manag. J. 2016, 37, 1472-1492. [CrossRef]

48. Dow, S.; McGuire, J. Propping and tunneling: Empirical evidence from Japanese keiretsu. J. Bank. Finance 2009, 33, 1817-1828. [CrossRef]

49. Hoshi, T.; Kashyap, A. Corporate Financing and Governance in Japan: The Road to the Future; MIT Press: Cambridge, MA, USA, 2001.

50. Morck, R.; Nakamura, M. Banks and Corporate Control in Japan. J. Finance 1999, 54, 319-339. [CrossRef]

51. Morck, R.; Nakamura, M.; Shivdasani, A. Banks, ownership structure, and firm value in Japan. J. Bus. 2000, 73, 539-567. [CrossRef]

52. Hiraki, T.; Inoue, H.; Ito, A.; Kuroki, F.; Masuda, H. Corporate governance and firm value in Japan: Evidence from 1985 to 1998. Pacific-Basin Finance J. 2003, 11, 239-265. [CrossRef]

53. Lincoln, J.R.; Gerlach, M.L.; Takahashi, P. Keiretsu Networks in the Japanese Economy: A Dyad Analysis of Intercorporate Ties. Am. Sociol. Rev. 1992, 57, 561. [CrossRef]

54. Kim, H.; Hoskisson, R.E.; Wan, W.P. Power dependence, diversification strategy, and performance in keiretsu member firms. Strat. Manag. J. 2004, 25, 613-636. [CrossRef]

55. McGuire, J.; Dow, S. The persistence and implications of Japanese keiretsu organization. J. Int. Bus. Stud. 2003, 34, 374-388. [CrossRef]

56. Brouthers, L.E.; Gao, Y.; Napshin, S. Keiretsu centrality-profits and profit stability: A power dependence perspective. J. Bus. Res. 2014, 67, 2603-2610. [CrossRef] 
57. Financial Service Agency. Japan's Stewardship Code. 2014. Available online: https://www.fsa.go.jp/en/refer/ councils/stewardship/20170529/01.pdf (accessed on 18 August 2019).

58. Financial Service Agency. Japan's Corporate Governance Code. 2015. Available online: https://www.fsa.go. jp/en/refer/councils/corporategovernance/20150306-1/01.pdf (accessed on 18 August 2019).

59. Shleifer, A.; Vishny, R.W. Large Shareholders and Corporate Control. J. Politi- Econ. 1986, 94, 461-488. [CrossRef]

60. Agrawal, A.; Mandelker, G.N. Large Shareholders and the Monitoring of Managers: The Case of Antitakeover Charter Amendments. J. Financial Quant. Anal. 1990, 25, 143. [CrossRef]

61. Brickley, J.A.; Lease, R.C.; Smith, C.W. Ownership structure and voting on antitakeover amendments. J. Financial Econ. 1988, 20, 267-291. [CrossRef]

62. Jarrell, G.A.; Poulsen, A.B. Shark repellents and stock prices: The effects of antitakeover amendments since 1980. J. Financ. Econ. 1987, 19, 127-168. [CrossRef]

63. Aguilera, R.V.; Desender, K.; López-Puertas Lamy, M.; Lee, J.H. The governance impact of a changing investor landscape. J. Int. Bus. Studies 2017, 48, 195-221. [CrossRef]

64. La Porta, R.; Lopez-De-Silanes, F.; Shleifer, A.; Vishny, R.W. Agency Problems and Dividend Policies around the World. J. Finance 2000, 55, 1-33. [CrossRef]

65. Abegglen, J.C.; Stalk, G. Kaisha, the Japanese Corporation; Basic Books: New York, NY, USA, 1985.

66. Blinder, A.S. More like them? Am. Prospect 1992, 3, 51-62.

67. Uno, J.; Kamiyama, N. Ownership structure, liquidity, and firm value: Effects of the investment horizon. Presented at the 22nd Australian Banking \& Finance Conference, Sydney, Austrilia, 16-18 December 2009.

68. Nguyen, P. The impact of foreign investors on the risk-taking of Japanese firms. J. Jpn. Int. Econ. 2012, 26, 233-248. [CrossRef]

69. Ikeda, N.; Inoue, K.; Watanabe, S. Enjoying the quiet life: Corporate decision-making by entrenched managers. J. Jpn. Int. Econ. 2018, 47, 55-69. [CrossRef]

70. Motta, E.M.; Uchida, K. Institutional investors, corporate social responsibility, and stock price performance. J. Jpn. Int. Econ. 2018, 47, 91-102. [CrossRef]

71. Shareholders wary of contrived defences. Financial Times, 22 May 2005.

72. TSE plans crackdown on groups that abuse minority investor rights. Financial Times, 31 July 2007.

73. Subsidiaries in Japan. Financial Times, 16 August 2007.

74. Sakawa, H.; Watanabel, N. Parent control and ownership monitoring in publicly listed subsidiaries in Japan. Res. Int. Bus. Finance 2018, 45, 7-14. [CrossRef]

75. Corporate Japan needs to ditch unnecessary 'poison pills'. Financial Times, 28 May 2018.

76. Leading critic of Japan Inc puts funds on alert. Financial Times, 11 June 2018.

77. Yermack, D. Higher market valuation of companies with a small board of directors. J. Financial Econ. 1996, 40, 185-211. [CrossRef]

78. Sakawa, H.; Watanabel, N. Board structures and performance in the banking industry: Evidence from Japan. Int. Rev. Econ. Finance 2018, 56, 308-320. [CrossRef]

79. Wooldridge, J.M. Econometric Analysis of Cross Section and Panel Data; MIT Press: Cambridge, MA, USA, 2002.

80. Cheung, S. Board size and the variability of corporate performance. J. Financ. Econ. 2008, 87, 157-176. [CrossRef]

81. Ferreira, M.A.; Matos, P. The colors of investors' money: The role of institutional investors around the world. J. Financial Econ. 2008, 88, 499-533. [CrossRef]

82. Kang, J.-K.; Stulz, R. Why is there a home bias? An analysis of foreign portfolio equity ownership in Japan. J. Financial Econ. 1997, 46, 3-28. [CrossRef]

83. Rediker, K.J.; Seth, A. Boards of directors and substitution effects of alternative governance mechanisms. Strat. Manag. J. 1995, 16, 85-99. [CrossRef]

(C) 2020 by the authors. Licensee MDPI, Basel, Switzerland. This article is an open access article distributed under the terms and conditions of the Creative Commons Attribution (CC BY) license (http://creativecommons.org/licenses/by/4.0/). 